\title{
Realization of Self-Preserving Size Distribution Theory for the Evolution of Tropospheric Atmospheric Aerosols through an Inverse Gaussian Distribution
}

Shen, J.

2021-07-01

Shen , J , Yu , M , Koivisto , A J , Jiang , H , Liu , Y , Wang , L \& Hussein , T 2021 , '

Realization of Self-Preserving Size Distribution Theory for the Evolution of Tropospheric

Atmospheric Aerosols through an Inverse Gaussian Distribution ', Journal of the

Atmospheric Sciences , vol. 78 , no. 7 , pp. 2143-2160 . https://doi.org/10.1175/JAS-D-20-0273.1

http://hdl.handle.net/10138/338162

https://doi.org/10.1175/JAS-D-20-0273.1

unspecified

publishedVersion

Downloaded from Helda, University of Helsinki institutional repository.

This is an electronic reprint of the original article.

This reprint may differ from the original in pagination and typographic detail.

Please cite the original version. 


\title{
Realization of Self-Preserving Size Distribution Theory for the Evolution of Tropospheric Atmospheric Aerosols through an Inverse Gaussian Distribution
}

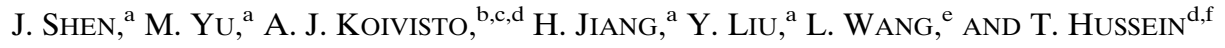 \\ ${ }^{a}$ Laboratory of Aerosol Science and Technology, China Jiliang University, Hangzhou, China \\ ${ }^{\mathrm{b}}$ ARCHE Consulting, Wondelgem, Belgium \\ ${ }^{\mathrm{c}}$ Air Pollution Management, Copenhagen, Denmark \\ ${ }^{\mathrm{d}}$ Institute for Atmospheric and Earth System Research (INAR), University of Helsinki, Helsinki, Finland \\ ${ }^{\mathrm{e}}$ Department of Environmental Science and Engineering, Fudan University, Shanghai, China \\ ${ }^{\mathrm{f}}$ Department of Physics, School of Science, University of Jordan, Amman, Jordan
}

(Manuscript received 12 September 2020, in final form 21 April 2021)

\begin{abstract}
The inverse Gaussian distributed method of moments (IGDMOM) was developed to analytically solve the kinetic collection equation (KCE) for the first time. Using the IGDMOM, we obtained both new analytical and asymptotic solutions to the KCE. This is shown for both the free-molecular and continuum regime collision frequency functions. The new analytical solutions are highly suitable for demonstrating the self-preserving size distribution (SPSD) theory. The SPSD theory is considered one of the most elegant research works in atmospheric science for aerosols or small cloud droplets. It was initially discovered by Friedlander and then developed by Lee with an assumption of the time-dependent lognormal size distribution function. In this study, we demonstrate that the SPSD theory of coagulating atmospheric aerosols can be presented in a simpler and more rigorous theoretical way, which is realized through the introduction of the IGDMOM for describing aerosol size distributions. Using the IGDMOM, the new formulas for the SPSD, as well as the time required for aerosols to reach the SPSD, are analytically provided and verified. Furthermore, we discover that the SPSD of atmospheric aerosols undergoing coagulation is only determined using a shape factor variable $\Omega$, which is composed of the first three moments at an initial stage. This study has critical implications for developing tropospheric atmospheric aerosol or small cloud droplet dynamics models and further verifies the SPSD theory from the viewpoint of theoretical analysis.
\end{abstract}

KEYWORDS: Atmosphere; Numerical analysis/modeling; Model comparison; Aerosols/particulates

\section{Introduction}

In the study of atmospheric aerosols, a major discovery was that with time, tropospheric aerosols might lose their "birthmarks" and acquire a size distribution independent of the physical properties of the medium (e.g., temperature, viscosity, and density) and time (Pruppacher and Klett 1997; Clark and Whitby 1967). This phenomenon puzzled scientists in the first half of the last century before Friedlander established the selfpreserving size distribution (SPSD) theory (Friedlander 1960) to research turbulence; here, the SPSD was attributed to aerosol coagulation, resulting in a shift from small aerosol condensation nuclei to the Aitken range in the upper end of the spectrum.

In the SPSD theory (Swift and Friedlander 1964; Friedlander and Wang 1966), a similarity transformation method (STM) was introduced to obtain asymptotic solutions to the kinetic collection equation (KCE). The STM assumes that particle size distribution (PSD) is a function of only particle volume normalized by the average particle volume. Substituting Eq. (28) into Eq. (1) with different coagulation kernels for the continuum and free-molecular regimes yields ordinary integrodifferential equations for $\psi$, with $\eta$ the independent variable after some complex processing; however, analytical

Corresponding author: Mingzhou Yu, yumingzhou1738@ yahoo.com solutions to the ordinary integrodifferential equations can only be found for the upper and lower ends of the distribution by making suitable approximation (Friedlander 2000), or numerical results can be obtained using numerical approaches such as the finite-difference method, the detailed numerical process can be found in Friedlander and Wang (1966).

Lee (1983) further developed the SPSD theory based on the concept of the method of moments (MOM) by presuming that size distribution during the coagulation process can be represented by a time-dependent lognormal function (Lee 1983; Lee et al. 1984; Park and Lee 2001; Wang et al. 2019). This method is called " $\log$ MOM" in the present article. The SPSD function $\psi(\eta)$ presented by $\log$ MOM was obtained by combining Eq. (28) and the lognormal distribution function $n(v, t)$, in which the parameters with respect to time will approach their limit values when time tends to infinity; thus, the analytical expression of the parameters should be obtained first. The SPSD theory via $\log$ MOM not only avoids the difficulty of solving the ordinary integrodifferential equations in the STM but directly yields the analytical expressions of SPSD. Furthermore, the analytical solutions to the KCE provided by $\log$ MOM are more accurate than those through the STM by Friedlander (Lee 1983).

Since these findings, numerous studies have developed and tested the SPSD theory (Dekkers and Friedlander 2002; Hidy 1965; Junge 1969; Lai et al. 1972; Liu and Whitby 1968; Nathans et al. 1970; Frenklach 1985). Typically, the SPSD theory has 
played a major role in explaining particle coagulation in gases, liquids, and recently gelation (Goudeli et al. 2015; Park et al. 2000; Söderlund et al. 1998; Zhao et al. 2015). This theory has also been used to explain particle evolution after nuclear air bursts and aerosol distribution in the urban atmosphere; size distribution calculated based on the SPSD theory was consistent with that obtained through experimental procedures (Clark and Whitby 1967; Nathans et al. 1970). Moreover, based on the SPSD theory, analytical solutions to the KCE can be obtained, which is a logical procedure that yields a relatively accurate solution and has strict functional forms in explicit expressions, from which any corresponding value associated with distribution function (such as $k$ th moment, mean, variance, kurtosis, skewness) can be calculated. Because of their generality, analytical solutions can intuitively reflect the relationships among various aerosol mechanisms and can be used for both qualitative and quantitative analyses (Turco and Yu 1998, 1997; Kelesidis and Goudeli 2021; Hussein et al. 2020, 2004; Ensor 2011).

The lognormal distribution was introduced to the description of dispersed particles by Kolmogorov (1941) and was introduced to the field of atmospheric science by Foitzik (1965) for describing optical aerosol properties (Kolmogorov 1941; Foitzik 1965). With its extended methods, the lognormal distribution has been the most widely used function for characterizing atmospheric aerosol size distribution (Cai et al. 2016; Riemer et al. 2009; Wang et al. 2012; Wang et al. 2019). Recently, a three-parameter inverse Gaussian distribution (IGD) was demonstrated to be a more suitable function than the lognormal distribution for characterizing atmospheric aerosol size distribution, and a new MOM, namely, the inverse Gaussian distributed MOM (IGDMOM), was established for solving the $\mathrm{KCE}$ involving Brownian coagulation, turbulent shear coagulation, and condensation (Shen et al. 2020b). IGD dates back to 1915 when Schrödinger (1915) and Smoluchowski (1915) presented independent derivations of the density of the first passage time distribution of Brownian motion with positive drift (Schrödinger 1915; Smoluchowski 1915). The modern statistical community was acquainted with this law by the pioneering work of Tweedie (1957). Seshadri (1999) provided a general discussion of the early history; later, IGD was first used to describe the motion of pollen particles in water as well as lifetime distribution by (Chhikara and Folks 1977), who elaborates the advantages in the use of the inverse Gaussian over the lognormal. To the best of our knowledge, adopting the generalized IGD as an aerosol/cloud PSD model was first proposed by Alexandrov and Lacis (2000) to characterize aerosol radiative properties. We later observed that the IGDMOM can characterize the skewness and kurtosis of size distribution more accurately than the log MOM (Shen et al. 2020b).

This study derived explicit analytical solutions with the realistic collision kernel for aerosol particle coagulation in the continuum and free-molecular regimes. In the following sections, the analytical solutions of the parameters in the IGD with respect to time are presented by rigorous mathematics derivation; the asymptotic expressions of the zeroth moment, second moment, and shape factor are then obtained as time tends to infinity; and finally, the analytical expressions of the SPSD through the IGDMOM and the function of time required to reach the SPSD are both determined theoretically. Compared with the other methods, including recognizable STM and $\log$ MOM, the SPSD obtained by the IGDMOM has the advantages of logical and rigorous derivation process, concise analysis expression, and competitive precision. Noted here only coagulation is considered in the present study because other dynamics, such as condensation, might make the SPSD nonexistent, which is shown in appendix G. Notably, all nomenclature, Greek letters, and abbreviations appearing in this article are presented in appendix $\mathrm{H}$.

\section{Theory}

\section{a. Numerical solution to KCE using the IGDMOM}

The KCE has long been used to mathematically model the time evolution of size distributions of cloud droplets and aerosols due to their collision-coalescence events (Wang et al. 2007). The KCE undergoing coagulation can be described using the following integrodifferential expression (Müller 1928; Bott 2000; Scott 1968; Long 1974):

$$
\begin{aligned}
\frac{\partial n(v, t)}{\partial t}= & \underbrace{\frac{1}{2} \int_{0}^{v} \beta\left(v-v_{1}, v_{1}\right) n\left(v-v_{1}, t\right) n\left(v_{1}, t\right) d v_{1}}_{\text {Gain of aerosol with volume } v} \\
& \underbrace{-n(v, t) \int_{0}^{\infty} \beta\left(v, v_{1}\right) n\left(v_{1}, t\right) d v_{1}}_{\text {Loss of aerosol with volume } v}
\end{aligned}
$$

where $n(v, t)$ is the particle number concentration density for particle volume $v$ at time $t$, and $\beta\left(v, v_{1}\right)$ is the coagulation kernel for two particles with volume $v$ and $v_{1}$. The first term on the right-hand side of Eq. (1) is the gain integral, representing the production of particles with volume $v$ due to all possible binary collision events of small particles. The second term is the loss integral resulting from collision events involving particles with volume $v$ as source particles. The KCE is also known as the population balance equation, Smoluchowski equation, general dynamic equation, or stochastic coalescence equation (Wang et al. 2007). The complete solutions to Eq. (1) for realistic collision kernel do not exist due to the highly nonlinear integrodifferential equation itself and particle size dependency of the collision coefficient $\beta\left(v, v_{1}\right)$ (Valioulis and List 1984; Klett 1975; Khvorostyanov and Curry 2008; Scott 1968; Turco and Yu 1998; Lai et al. 1972).

Here, we present a brief discussion of the IGDMOM, which is used to solve Eq. (1) numerically in the framework of the MOM. The detailed derivation for the IGDMOM is presented in Shen et al. (2020b). Without loss of generality, the moment transformed equations of the SPSD can be obtained by multiplying Eq. (1) by $v^{k}$ and integrating over the entire size distribution (Lee et al. 1984):

$$
\begin{aligned}
\frac{d M_{k}}{d t}= & \frac{1}{2} \int_{0}^{\infty} \int_{0}^{\infty}\left[\left(v+v_{1}\right)^{k}-v^{k}-v_{1}^{k}\right] \\
& \times \beta\left(v, v_{1}\right) n(v, t) n\left(v_{1}, t\right) d v d v_{1}, \quad k=0,1,2, \ldots,
\end{aligned}
$$

where the $k$ th moment of the size distribution is defined as follows: 


$$
M_{k}=\int_{0}^{\infty} v^{k} n(v, t) d v
$$

To solve Eq. (3), we apply a time-dependent IGD to approximate the density function $n(v, t)$. IGD has the following probability density function (Folks and Chhikara 1978):

$$
n(v, t)=N(t) \sqrt{\frac{\varphi(t)}{2 \pi v^{3}}} e^{-\varphi(t)[v-\phi(t)]^{2} /\left[2 v \phi^{2}(t)\right]},
$$

where $N(t)$ is the total particle number concentration, and IGD is characterized by three positive parameters, $N, \varphi$, and $\phi$. For $v>0, \phi(t)$ is the mean of the distribution, and $\varphi(t)$ is the shape parameter. As $\varphi(t)$ tends to approach infinity, IGD approximates a Gaussian distribution. Notably, Alexandrov and Lacis (2000) provide the general form of Eq. (4). Substituting Eq. (4) into Eq. (3) gives its moment closure:

$$
M_{k}=N(t) \sqrt{\frac{2}{\pi}} s^{1 / 2} e^{s} \phi^{k} K_{k-1 / 2}(s),
$$

where $K_{k-1 / 2}(s)$ is the second type of modified Bessel function. Let $s=\varphi / \phi$, then the parameters can be expressed using the first three moments as follows:

$$
\phi=\frac{M_{1}}{M_{0}}
$$

and

$$
s=\frac{M_{1}^{2}}{M_{0} M_{2}-M_{1}^{2}} .
$$

For atmospheric aerosol or cloud droplet coagulation due to Brownian motion in the continuum regime, the collision kernel $\beta\left(v, v_{1}\right)$ is given by Smoluchowski (1917):

$$
\beta\left(v, v^{\prime}\right)=K_{\mathrm{CO}}\left(\frac{1}{v^{1 / 3}}+\frac{1}{v^{1 / 3}}\right)\left(v^{1 / 3}+v^{1 / 3}\right)
$$

where $K_{\mathrm{CO}}=2 k_{B} T /(3 \mu)$ is the coagulation coefficient for the continuum regime, $k_{B}$ is the Boltzmann constant, $T$ is the absolute gas temperature, and $\mu$ is the gas viscosity. The continuum theory can be applied to atmospheric particles larger than a few tenths of a micron (Lai et al. 1972).

The collision kernel, $\beta\left(v, v_{1}\right)$, in the free-molecular regime, is given as follows (Friedlander 2000):

$$
\beta\left(v, v^{\prime}\right)=K_{\mathrm{FM}}\left(\frac{1}{v}+\frac{1}{v^{\prime}}\right)^{1 / 2}\left(v^{1 / 3}+v^{\prime 1 / 3}\right)^{2},
$$

where $K_{\mathrm{FM}}=[3 /(4 \pi)]^{1 / 6}\left(6 k_{B} T / \rho\right)^{1 / 2}$ is the coagulation coefficient for the free-molecular regime and $\rho$ is the mass density of the particle.

According to the IGDMOM (Shen et al. 2020b), the evolution equations of moments for Brownian coagulation can be expressed for the continuum regime collision frequency function as follows:

$$
\left\{\begin{array}{c}
\frac{d M_{0}}{d t}=-K_{\mathrm{CO}} M_{1}^{2} \frac{2}{\pi} s e^{2 s} \phi^{-2}\left\{\left[K_{1 / 2}(s)\right]^{2}+K_{1 / 6}(s) K_{5 / 6}(s)\right\}, \\
\frac{d M_{1}}{d t}=0, \\
\frac{d M_{2}}{d t}=2 K_{\mathrm{CO}} M_{1}^{2} \frac{2}{\pi} s e^{2 s}\left\{\left[K_{1 / 2}(s)\right]^{2}+K_{1 / 6}(s) K_{5 / 6}(s)\right\} .
\end{array}\right.
$$

The evolution equations for the free-molecular regime collision frequency function can be expressed as follows:

$$
\left\{\begin{array}{c}
\frac{d M_{0}}{d t}=-\sqrt{2} K_{\mathrm{FM}} b_{0} M_{1}^{2} \frac{2}{\pi} s e^{2 s} \phi^{-11 / 6}\left\{K_{1 / 12}(s) K_{3 / 4}(s)+\left[K_{5 / 12}(s)\right]^{2}\right\} \\
\frac{d M_{1}}{d t}=0 \\
\frac{d M_{2}}{d t}=2 \sqrt{2} K_{\mathrm{FM}} b_{2} M_{1}^{2} \frac{2}{\pi} s e^{2 s} \phi^{1 / 6}\left\{K_{11 / 12}(s) K_{1 / 4}(s)+\left[K_{7 / 12}(s)\right]^{2}\right\}
\end{array}\right.
$$

The method for obtaining $b_{0}$ and $b_{2}$ in Eq. (11), as well as their expressions, are presented in appendix A.

\section{b. Analytical solutions to the KCE}

The study of the analytical solution to the KCE is not new; Smoluchowski (1917) was the first to provide an analytical expression for the early coagulation stage of an aerosol consisting of monodisperse aerosols, and his work was subsequently developed by Friedlander (1960), Lee et al. (Lee et al. 1990; Lee 1983), and Yu et al. (2016, 2015a). The detailed mathematical expressions for these analytical solutions are presented in appendix B.

Other works on the analytical solutions to the KCE include the study of cloud droplet coalescence in which Scott (1968) solved the KCE analytically using three types of unrealistic collection kernels. In the study of the steady-state, sourceenhanced atmospheric aerosol, Klett obtained the analytical solution to the KCE by assuming the collection kernel $K=$ $K_{0} u^{\alpha} v^{\alpha}$, where $K_{0}$ and $\alpha$ are constants (Klett 1975). Moreover, the analytical solutions to the KCE were executed considering plume expansion, condensation, and nucleation by Turco et al. (Turco and Yu 1997, 1998, 1999; Yu and Turco 1998) and puff/plume coagulation effects in the presence of atmospheric dispersion by Anand and Mayya (2011); the collision formulas in these studies are simplified to be constant or reduced forms to include maximum dynamics.

In the present study, however, the analytical solution to the $\mathrm{KCE}$ with the realistic collision kernel for Brownian 
coagulation is considered by implementing the IGDMOM. For simplification, other dynamical processes such as dispersion, condensation, and nucleation are not included. Because this paper focuses on the SPSD of coagulation, the analytical solutions discussed in this section are mainly to derive the final expressions of SPSD, whereas kinetic processes are not required here, and they can be discussed separately in another article later. For further discussion, we consider the following definitions:

$$
\frac{M_{1}}{M_{0}}=E
$$

and

$$
\frac{M_{2} M_{0}}{M_{1}^{2}}=\Omega
$$

where $E$ is $\phi$, and $s=1 /(\Omega-1)$ for IGD based on Eqs. (6) and (7). Furthermore, $\Omega$ has the property $\Omega>1$ for $s>0$. Once $E$ and $\Omega$ are defined, we find that the $k$ th moments in various MOMs, including the IGDMOM, TEMOM, and log MOM, have the same closure function form:

$$
M_{k}=M_{0} E^{k} f(\Omega, k) .
$$

The derivations of Eq. (14) in terms of the IGDMOM, TEMOM, and $\log$ MOM are presented in appendix C. In the subsequent discussion, the shape factor $\Omega$ is found to be closely related to other vital characteristics of a PSD.

\section{1) BROWNiAN COAgULATION IN THE CONTINUUM REGIME}

According to the derivation presented in appendix D, Eq. (10) has its solution as follows:

$$
\left\{\begin{array}{c}
\frac{M_{0}}{M_{00}}=\frac{1}{[1+a(s) \tau]+1} \\
M_{1}=M_{10} \\
\frac{M_{2}}{M_{20}}=\frac{2}{\Omega_{0}}[1+a(s)] \tau+1
\end{array}\right.
$$

where $M_{00}, M_{10}, M_{20}$, and $\Omega_{0}$ are the initial values of $M_{0}, M_{1}$, $M_{2}$, and $\Omega$, and $\tau=K_{\mathrm{CO}} M_{00} t . a(s)$ is a monotonically decreasing function for $s>0$, whose expression and characteristic are presented in appendix D.

Substituting Eq. (15) into Eqs. (6) and (7), and combining Eq. (13), gives the following expressions:

$$
\phi=E=E_{0}\{[1+a(s)] \tau+1\}
$$

where $E_{0}$ is the initial value of $E$,

$$
s=1+\frac{2-\Omega_{0}}{[1+a(s)] \tau+\Omega_{0}-1}
$$

and

$$
\Omega=2+\frac{\Omega_{0}-2}{[1+a(s)] \tau+1} .
$$

Equation (18a) can be further written as follows:

$$
\frac{\Omega-2}{\Omega_{0}-2}=\frac{1}{[1+a(s)] \tau+1} .
$$

Notably, $s=1 /(\Omega-1)$. It also should be mentioned that $a(s)$ in Eqs. (15)-(18) can be assigned as the initial value $a\left(s_{0}\right)$ for simplified calculation, which has been discussed in appendix D.

Finally, the entire size distribution due to Brownian coagulation in the continuum regime at any time can be constructed using Eq. (4), with the values of $\phi$ and $s$ presented in Eqs. (16) and (17). In particular, when $\tau \rightarrow \infty$, we have $\Omega_{\infty}$ in Eq. (18a) and $s \rightarrow 1$ in Eq. (17). Simultaneously, the following results can be obtained:

$$
\lim _{\tau \rightarrow+\infty} a[s(\tau)]=a(1)=1.081442 .
$$

\section{2) BROWNIAN COAGULATION IN THE FREE- MOLECULAR REGIME}

According to the derivation presented in appendix E, the analytical solution of Eq. (11) can be written as follows:

$$
\left\{\begin{array}{c}
\frac{M_{0}}{M_{00}}=\left[\frac{5 \sqrt{2}}{6} a_{0}(s) b_{0}(s) \tau+1\right]^{-6 / 5}, \\
M_{1}=M_{10}, \\
\frac{M_{2}}{M_{20}}=\frac{1}{A \Omega_{0}}\left\{\left[\frac{5 \sqrt{2}}{6} a_{0}(s) b_{0}(s) \tau+1\right]^{6 / 5}+A \Omega_{0}-1\right\},
\end{array}\right.
$$

where $\tau=K_{\mathrm{FM}} E_{0}^{1 / 6} M_{00} t, a_{0}(s)$ and $a_{2}(s)$ are monotonically increasing and decreasing functions for $s>0$, respectively, whose expressions and characteristics are presented in appendix E, and

$$
A=\frac{a_{0}(s)}{2 a_{2}(s)} .
$$

Substituting Eq. (20) into Eqs. (6) and (7) gives

$$
\begin{aligned}
& \phi=E=E_{0}\left[\frac{5 \sqrt{2}}{6} a_{0}(s) b_{0}(s) \tau+1\right]^{6 / 5}, \\
& s=\frac{A}{\left(A \Omega_{0}-1\right)\left[\frac{5 \sqrt{2}}{6} a_{0}(s) b_{0}(s) \tau+1\right]^{-6 / 5}+1-A},
\end{aligned}
$$

and

$$
\Omega=\frac{1}{A}\left\{\left(A \Omega_{0}-1\right)\left[\frac{5 \sqrt{2}}{6} a_{0}(s) b_{0}(s) \tau+1\right]^{-6 / 5}+1\right\} .
$$

Equation (24a) can be further written as follows:

$$
\frac{\Omega-\frac{1}{A}}{\Omega_{0}-\frac{1}{A}}=\left[\frac{5 \sqrt{2}}{6} a_{0}(s) b_{0}(s) \tau+1\right]^{-6 / 5}
$$


Finally, the size distribution due to Brownian coagulation in the free-molecular regime at any time $\tau$ can be constructed using Eq. (4) with the values of $\phi$ and $s$ presented in Eqs. (22) and (23). As $\tau \rightarrow \infty$, we have

$$
s \rightarrow \frac{a_{0}(s)}{2 a_{2}(s)-a_{0}(s)}=\frac{A}{1-A} .
$$

The solution of the following equation:

$$
s=\frac{a_{0}(s)}{2 a_{2}(s)-a_{0}(s)}
$$

gives the limit value of $s=1.76034$, and then $\Omega \rightarrow 1 / A=$ $1 / s+1=2.31$, from which the constant $A$ can be defined to be equal to 0.4319 .

It must be mentioned that $a_{k}(s)$ and $b_{k}(s)(k=0,2)$ in Eqs. (20)-(24) can be assigned as the initial values $a_{k}\left(s_{0}\right)$ and $b_{k}\left(s_{0}\right)$ $(k=0,2)$ for simplified calculation, which has been discussed in appendix E.

Notably, benefiting from the unique characteristics of the $k$ th moment of the IGDMOM, the analytical solution can be obtained directly and simply from the ordinary differential equations of the first three moments, which is completely different from that of the other methods, namely, the STM by Friedlander, $\log$ MOM by Lee et al., and TEMOM by Yu et al. (Chen et al. 2014; Lee 1983; Lee et al. 1984, 1997; Yu et al. 2015a,b).

\section{3) SPSD FORMULAS}

In the SPSD theory by Friedlander (1960), the dimensionless particle volume $\eta$ and the size distribution density function $\psi$ are defined as follows:

$$
\eta=\frac{v}{\tilde{v}}
$$

and

$$
\psi=\frac{n(v, t) \tilde{v}}{N}
$$

where $\tilde{v}$ is the arithmetic mean particle volume and is defined as follows:

$$
\tilde{v}=\frac{V}{N}=\frac{M_{1}}{M_{0}}=\phi,
$$

where $V$ is the total particle volume and $N$ is the total particle number concentration, which are identical to $M_{0}$ and $M_{1}$, respectively. Next, the variable $v$ can be expressed by $\phi$ and $\eta$ :

$$
v=\phi \eta
$$

Therefore, the IGD presented in Eq. (4) can be converted to a self-preserving form as follows:

$$
\psi(\eta)=\sqrt{\frac{s_{\infty}}{2 \pi \eta^{3}}} \exp \left[-\frac{s_{\infty}(\eta-1)^{2}}{2 \eta}\right],
$$

where $s_{\infty}$ is the limit of $s$ when $\tau \rightarrow \infty$.
According to the discussion for Brownian coagulation in the continuum regime [i.e., Eq. (17)], we have $s_{\infty}=1$, and thus, the SPSD form of the IGDMOM in this regime can be written as

$$
\psi(\eta)=\sqrt{\frac{1}{2 \pi \eta^{3}}} \exp \left[-\frac{(\eta-1)^{2}}{2 \eta}\right] .
$$

Similarly, the SPSD form for Brownian coagulation in the free-molecular regime with $s_{\infty}=0.76034$ is

$$
\psi(\eta)=\sqrt{\frac{0.76034}{2 \pi \eta^{3}}} \exp \left[-\frac{0.76034(\eta-1)^{2}}{2 \eta}\right] .
$$

Equations (32) and (33) are the SPSD expressions proposed in this study for Brownian coagulation in the continuum and free-molecular regimes, which are verified by comparing with other general expressions, as detailed in section 4 .

\section{4) SELF-PRESERVING TIME $\tau_{\infty}$}

It is critical to know how long it takes to reach SPSD, that is, the self-preserving time (SPT) — as aerosols are polydisperse. The SPT strongly depends on the initial aerosol size distribution (Lee 1983; Vemury et al. 1994; Frenklach 1985). For the first time, Hidy and Lilly gave the SPT for an initially monodisperse aerosol undergoing Brownian coagulation in the continuum regime (Hidy and Lilly 1965; Hidy 1965). Lee et al. derived expressions of the SPT in both continuum and freemolecular regimes as a function of the initial geometric standard deviation of the PSD (Lee et al. 1990). They defined the SPT as the time required for the geometric standard deviation of the distribution to approach its SPSD value within $\pm 10 \%$. In this article, following Lee et al.'s definition of SPT, the expression of SPT with respect to $\Omega_{0}$ is given.

The shape factor $\Omega_{\infty}$ in the continuum regime is obviously equal to 2 from Eq. (18a), and then the SPT $\left(\Omega_{\infty}=M_{00} K_{\mathrm{CO}} t_{\infty}\right)$ in the continuum regime is defined by

$$
\left|\Omega\left(\tau_{\infty}\right)-2\right|<0.1
$$

where $\tau_{\infty}$ can be obtained by substituting Eq. (34) into Eq. (18a):

$$
\tau_{\infty}=\left\{\begin{array}{ll}
\frac{-10\left(\Omega_{0}-2\right)-1}{1+a\left(\frac{1}{\Omega_{0}-1}\right)}, & 0<\Omega_{0}<1.9 \\
\frac{10\left(\Omega_{0}-2\right)-1}{1+a\left(\frac{1}{\Omega_{0}-1}\right)}, & \Omega_{0}>2.1 \\
0, & 1.9 \leq \Omega_{0} \leq 2.1
\end{array} .\right.
$$

In the free-molecular regime, $\Omega_{\infty}=2.3152$ as given in Eq. (24a), then the dimensionless SPT $\left(\tau_{\infty}=K_{\mathrm{FM}} E_{0}^{1 / 6} M_{00} t_{\infty}\right)$ in the free-molecular regime is defined as

$$
\left|\Omega\left(\tau_{\infty}\right)-2.3152\right|<0.1
$$

Next, $\tau_{\infty}$ can be obtained by substituting Eq. (36) into Eq. (24a): 


$$
\tau_{\infty}= \begin{cases}\frac{\left[-10\left(\Omega_{0}-2.3152\right)\right]^{5 / 6}-1}{\frac{5 \sqrt{2}}{6} a_{0}\left(\frac{1}{\Omega_{0}-1}\right) b_{0}\left(\frac{1}{\Omega_{0}-1}\right)}, & 0<\Omega_{0}<2.2152 \\ \frac{\left[10\left(\Omega_{0}-2.3152\right)\right]^{5 / 6}-1}{5 \sqrt{2}} a_{0}\left(\frac{1}{\Omega_{0}-1}\right) b_{0}\left(\frac{1}{\Omega_{0}-1}\right), & \Omega_{0}>2.4152 \\ 0, & 2.2152 \leq \Omega_{0} \leq 2.4152 .\end{cases}
$$

From Eqs. (35) and (37), the SPT can be determined based on only the initial shape factor; that is, $\Omega_{0}$, whereas Lee et al. reported that the SPT is determined based on only the initial geometric standard deviation $\sigma_{g 0}$ of SPD (Lee et al. 1990). The relation between $\Omega_{0}$ and $\sigma_{g 0}$ is presented in Eq. (47).

\section{Numerical implementation}

To verify the obtained analytical solutions to the $\mathrm{KCE}$ as well as SPSD expressions, we compared the results with highly recognizable schemes (Friedlander 1960, 1961; Lee 1983; Smoluchowski 1917; Yu et al. 2015a). Furthermore, the results were compared with a prestigious method, namely, the sectional method (SM; Landgrebe and Pratsinis 1990), which does not assume a particular size distribution during coagulation and has been extensively used to validate other methods as a reference (Lee 1983; Lee et al. 1984, 1990; Otto et al. 1999; Yu and Lin 2009a,b, 2017, 2018; Wang et al. 2019). In this paper, the SM is also used as a reference to validate the method proposed in this study. To ensure high numerical accuracy of this SM method, the section number is 800 and the section spacing factor is 1.045 (Shen et al. 2020a).

Here, to discover the ability of the analytical and asymptotic schemes proposed in this study to characterize the evolution of atmospheric aerosol and warm cloud droplets under high concentration conditions (Otto et al. 1999), the following initial parameters for the comparison calculations were chosen:

$$
\begin{aligned}
& N_{0}=1 \times 10^{16} \mathrm{~m}^{-3}, \\
& \rho_{p}=1000.0 \mathrm{~kg} \mathrm{~m}^{-3}, \\
& T=300 \mathrm{~K}, \\
& \mu=1.83 \times 10^{-5} \mathrm{~kg} \mathrm{~s}^{-1} \mathrm{~m}^{-1}, \\
& d_{p 0}=1.0 \mathrm{~nm} \text { (Brownian coagulation in the free-molecular } \\
& \text { regime), } \\
& d_{p 0}=0.5 \mu \mathrm{m} \text { (Brownian coagulation in the continuum } \\
& \text { regime), } \\
& \left.\sigma_{g 0}=1.2 \text { [i.e., } \Omega_{0}=e^{9 \mathrm{ln}^{2}(1.2)}=1.349\right] .
\end{aligned}
$$

The total particle number concentration, second moment, and shape factor are monitored as a function of time. The dimensionless moments are defined as $M_{k} / M_{k 0}$, and the dimensionless time is defined as $\tau=K_{\mathrm{CO}} M_{00} t$ in the continuum regime and $\tau=K_{\mathrm{FM}} E_{0}^{1 / 6} M_{00} t$ in the free-molecular regime. Here, $M_{\mathrm{k} 0}$ represents initial $k$ th moments at time $t=0$, which is defined as

$$
M_{k 0}=\chi^{k^{* k}}
$$

where $\chi=e^{\left(3 \ln \sigma_{g 0}\right)^{2} / 2}$, and $\sigma_{g 0}$ is the initial geometric standard deviation of aerosols. In this study, $\sigma_{g 0}=1.20$ for all investigated cases.

The definition for $M_{k 0}$ presented in Eq. (38) is derived from the work of Lee (1983), who assumed the atmospheric aerosol size distribution to be a lognormal size distribution, which was also used by Pratsinis (1988) and our previous study (Shen et al. 2020 b). For convenient analysis, all the quantities presented in section 4 , including total particle number concentration and the second moment, are presented in dimensionless form.

This study verified the precision of analytical solutions to the $\mathrm{KCE}$ as well as other mathematical expressions. To achieve this, the IGDMOM, log MOM, TEMOM, and SM were applied to solve the same PBE under the same conditions. The relative errors of the $k$ th moments of the investigated methods of moments to the SM are expressed as

$$
\text { Error }=\frac{M_{k}(\mathrm{MOM})-M_{k}(\mathrm{SM})}{M_{k}(\mathrm{SM})},
$$

where $M_{k}(\mathrm{MOM})$ is the $k$ th moment obtained from the investigated $\mathrm{MOM}$ and $M_{k}(\mathrm{SM})$ is the corresponding moments obtained from the referenced SM. All numerical calculations are implemented using the fourth-order Runge-Kutta method with a fixed time step of 0.001 in dimensionless form.

\section{Results and discussion}

By applying the IGD to the description of atmospheric aerosol size distribution, we noted that Friedlander's SPSD theory of coagulating atmospheric aerosols can be efficiently presented, which is reflected in the following four analytical solutions to the SCE, asymptotic analysis, SPT, and dimensionless self-preserving size distribution expression.

\section{a. Analytical solutions to the SCE}

Figure 1 illustrates the variance of the dimensionless total particle number concentration $M_{0} / M_{00}$ (Fig. 1a), the dimensionless second moment $M_{2} / M_{20}$ (Fig. 1b), and the shape factor $\Omega$ (Fig. 1c), with time as well as the relative errors of such quantities to the referenced SM (Figs. 1d-f) for Brownian coagulation in the continuum regime. In the figure, the analytical solutions to the KCE proposed by Smoluchowski (1917), Friedlander and Wang (1966), Lee (1983), and Yu et al. (2015a) are implemented and are compared with the solutions presented in Eqs. (15) and (18a). In the analytical solutions of Smoluchowski (1917) and Friedlander and Wang (1966), no information regarding $M_{2} / M_{20}$ is provided; thus, no curves are available for their solutions in Figs. 1b, 1c, 1e, and 1f. As proposed in the literatures (Landgrebe and Pratsinis 1990; Yu and Lin 2009a), the initial distribution of all MOMs here is chosen as lognormal distribution.

The analytical solution of Friedlander and Wang (1966) produces the largest errors for $M_{0} / M_{00}$, followed by that of Smoluchowski (1917). The IGDMOM, log MOM, and TEMOM overlap, and it is difficult to distinguish them, even from the relative errors presented in Fig. 1d; the relative errors of these three solutions to the SM are less than those of solutions by Smoluchowski (1917) and Friedlander and Wang 

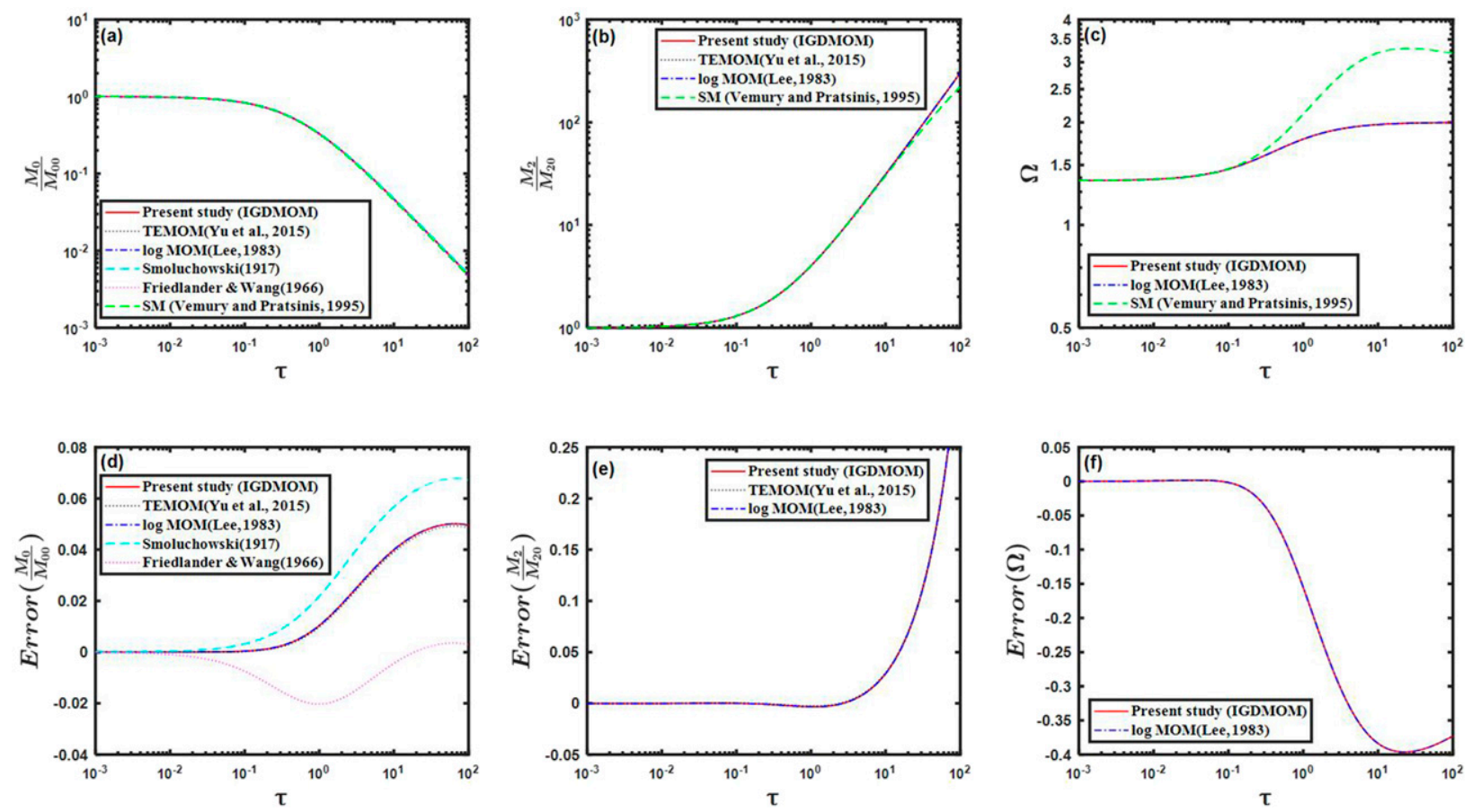

FIG. 1. Variances of dimensionless total particle number concentration $M_{0} / M_{00}$, dimensionless second moment $M_{2} / M_{20}$, and the shape factor $\Omega$ with time, as well as the relative errors of such quantities to the referenced SM for Brownian coagulation in the continuum regime. The scaled time is $\tau=K_{\mathrm{CO}} M_{00} t$.

(1966). This is because the solution by Smoluchowski accurately applies only to monodisperse aerosols, whereas the solution by Friedlander and Wang applies only to aerosols that have attained the SPSD distribution. For both $M_{2} / M_{20}$ and $\Omega$, Figs. 1b, 1c, 1e, and 1f illustrate that the present solution (IGDMOM) gives nearly the same results as that produced by the $\log$ MOM and TEMOM. Thus, the analytical solution proposed in this article has the same accuracy for characterizing the evolution of aerosol quantities, including $M_{0} / M_{00}$, $M_{2} / M_{20}$, and $\Omega$ as the $\log$ MOM and TEMOM, although they have different mathematical expressions.

Similarly, the analytical solutions to the KCE for Brownian coagulation in the free-molecular regime are displayed in Fig. 2 for the three typical quantities, namely, $M_{0} / M_{00}, M_{2} / M_{20}$, and $\Omega$, through the implementation of the present solution and solutions by Smoluchowski (1917), Lai et al. (1972), Lee et al. (1984), and Yu et al. (2015a). The present solutions, presented in Eqs. (20) and (24a), are used for the calculation. For the dimensionless total particle number concentration, $M_{0} / M_{00}$, the solution by Smoluchowski generates the largest errors relative to the SM. The present solution generates curves that overlap with the TEMOM and log MOM, whose relative errors are nearly the same as the solution by Lai et al. (1972), although the solution by Lai et al. (1972) theoretically only applies to aerosols that have attained the SPSD due to similarity transformation. The present solution is nearly the same as that by the TEMOM and log MOM for $M_{2} / M_{20}$, whereas for the shape factor $\Omega$ presented in Figs. 2c and 2f, the present solution produces significantly fewer errors than the log MOM.
In summary, through the examination of three typical quantities, namely, $M_{0} / M_{00}, M_{2} / M_{20}$, and $\Omega$, the analytical solutions proposed in this study have higher accuracy than solutions by Smoluchowski (1917) and other researchers (Friedlander and Wang 1966; Lai et al. 1972). The present solutions provide similar or higher accuracy than the log MOM for $M_{0} / M_{00}, M_{2} / M_{20}$, and $\Omega$, although all these solutions have different mathematical expressions and are obtained from different size distributions.

\section{b. Asymptotic analysis for SPSD}

\section{1) BROWNiAN COAGULATION IN THE CONTINUUM REGIME}

Dimensionless moments for the SPSD are an essential consideration for asymptotic analysis in the MOM. From Eq. (15), the time evolution of the dimensionless zeroth moment for the SPSD in the continuum regime is as follows:

$$
\left.\frac{M_{0}}{M_{00}}\right|_{\text {SPSD }}=\frac{1}{\left[1+a\left(\Omega_{\infty}\right)\right] \tau+1},
$$

where $M_{00}$ is the initial value of $M_{0}, \tau=M_{00} K_{\mathrm{CO}} t$, and $1+$ $a\left(\Omega_{\infty}\right)=2.0814$. The dimensionless zeroth moments for the SPSD for other methods have a similar expression as follows:

$$
\left.\frac{M_{0}}{M_{00}}\right|_{\mathrm{SPSD}}=\frac{1}{\alpha \tau+1} .
$$

The values of $\alpha$ given by Lee (1983) and Friedlander (2000) are 2.080 and 2.129, respectively. For Smoluchowski's discretized 

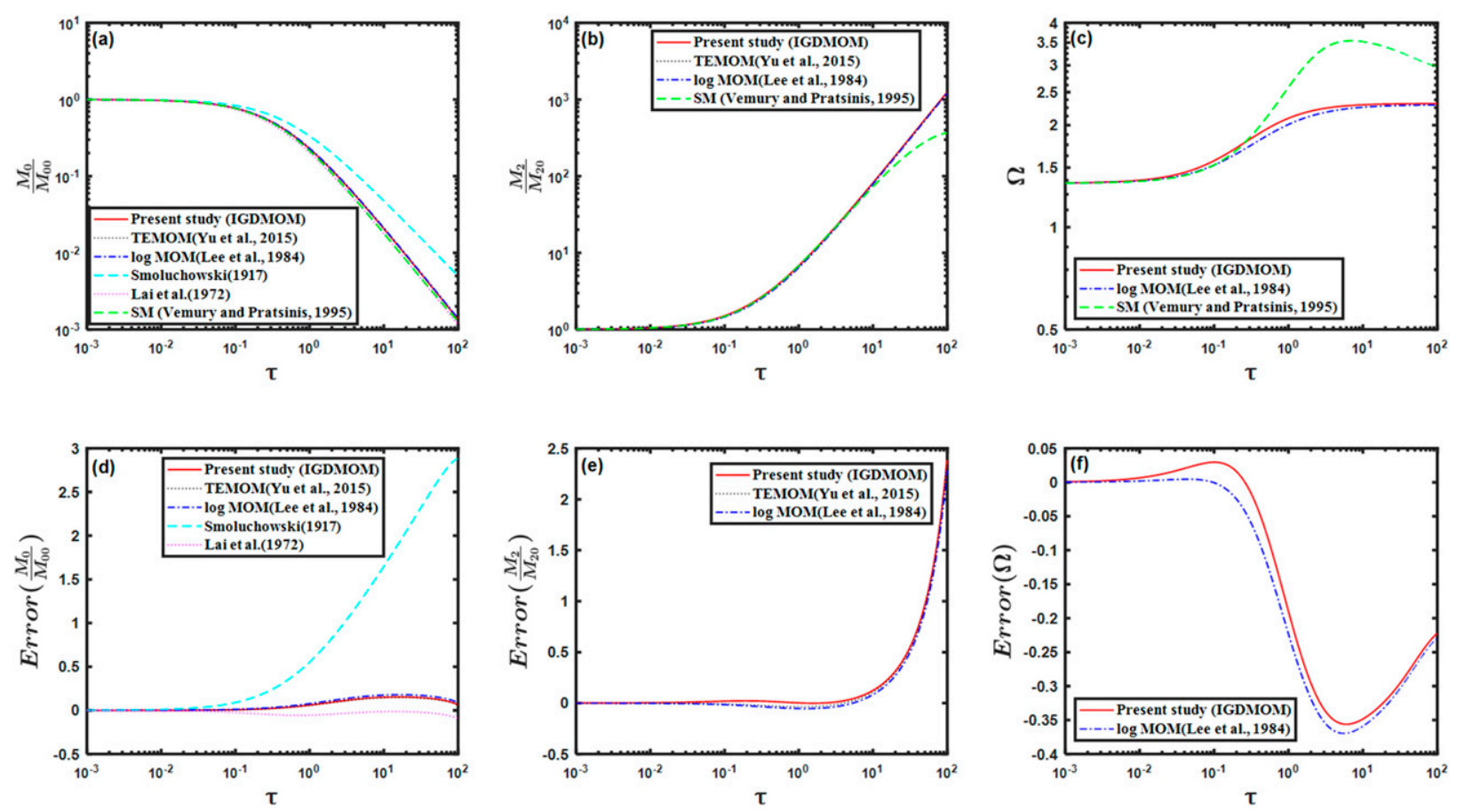

FIG. 2. Variances of dimensionless total particle number concentration $M_{0} / M_{00}$, dimensionless second moment $M_{2} / M_{20}$, and the shape factor $\Omega$ with time, as well as the relative errors of such quantities to the referenced SM for Brownian coagulation in the free-molecular regime. The scaled time is $\tau=K_{\mathrm{FM}} E_{0}^{1 / 6} M_{00} t$.

PSD with a constant collision kernel, the value of $\alpha$ is 2 (Smoluchowski 1917), whereas for the TEMOM it is 2.086 (Chen et al. 2014).

Figure 3a illustrates the evolution of the dimensionless zeroth moment predicted using different methods. All the results are in excellent agreement, especially those given by the present study and by Lee (1983) because the value of $\alpha$ given by these two methods is almost the same.

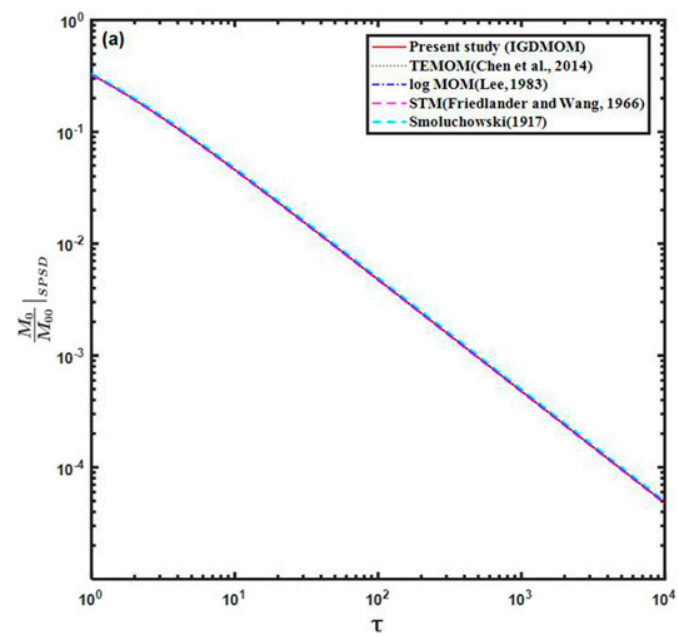

Moreover, assuming the size distribution has reached the SPSD in the continuum regime, the time evolution of the dimensionless second moment can be determined from Eq. (15):

$$
\left.\frac{M_{2}}{M_{20}}\right|_{\mathrm{SPSD}}=\varpi \tau+1,
$$

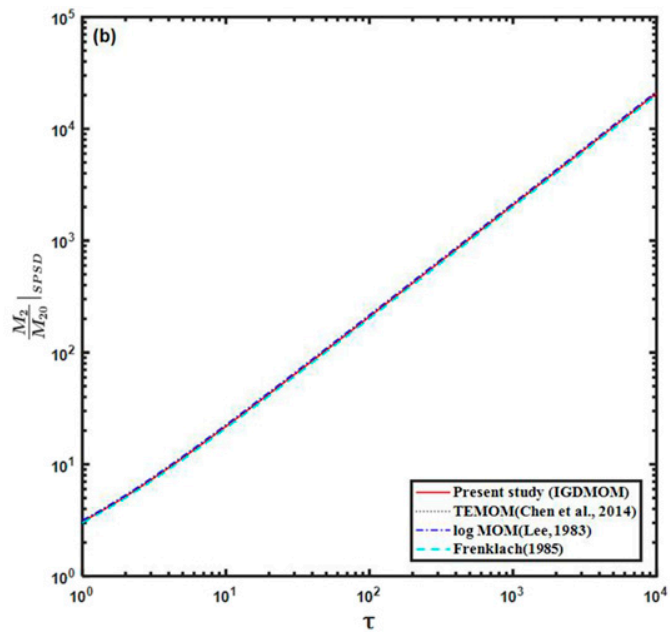

FIG. 3. Evolution of the dimensionless (a) zeroth moment and (b) second moment for the SPSD in the continuum regime. The scaled time is $\tau=K_{\mathrm{CO}} M_{00} t$. 

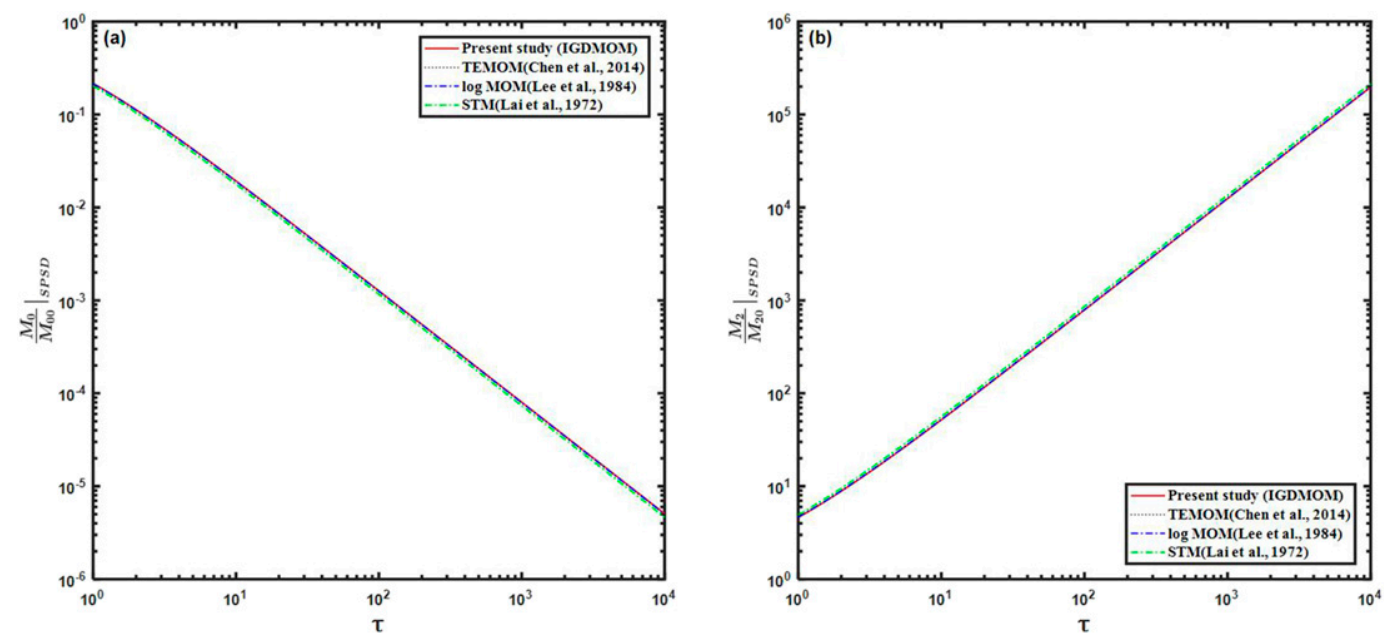

FIG. 4. Evolution of the dimensionless (a) zeroth moment and (b) second moment for the SPSD in the freemolecular regime. The scaled time is $\tau=K_{\mathrm{FM}} E_{0}^{1 / 6} M_{00} t$.

where $\varpi=1+a\left(\Omega_{\infty}\right)=2.0814$. Thus, the dimensionless second moment $M_{2} / M_{20}$ changes linearly with time. If the size distribution is specified as lognormal or is not presupposed as any distribution in the TEMOM, and is assumed to have achieved the SPSD, the time evolution of the second moment can be derived as $\left.\left(M_{2} / M_{20}\right)\right|_{\mathrm{SPSD}}=\varpi \cdot \tau+1$, where $\varpi=1+2^{1 / 9}=2.0801$ in the $\log$ MOM and $\varpi=338 / 162=2.0864$ in the TEMOM (Chen et al. 2014). For Smoluchowski's discretized size distribution with a constant collision kernel, $\varpi=2$ (Frenklach 1985). Figure $3 \mathrm{~b}$ illustrates the evolution of the dimensionless secondary moment predicted by different methods. The present IGDMOM, TEMOM, log MOM, and Frenklach's method produce overlapped curves, which cannot be distinguished from each other.

\section{2) BROWNiAN COAGULATION IN THE FREE- MOLECULAR REGIME}

Similar to the continuum regime, in the free-molecular regime, the shape factor $\Omega$ can be used to investigate the time evolution of the PSD in the SPSD. From Eq. (20), the time evolution of the dimensionless zeroth moment for the SPSD when $\Omega$ reaches $\Omega_{\infty}$ after a sufficient length of time in the freemolecular regime, is as follows:

$$
\left.\frac{M_{0}}{M_{00}}\right|_{\mathrm{SPSD}}=\left[\frac{5 \sqrt{2}}{6} a_{0}\left(\Omega_{\infty}\right) b_{0}\left(\Omega_{\infty}\right) \tau+1\right]^{-6 / 5},
$$

where $M_{00}$ is the initial value of $M_{0}, \tau=K_{\mathrm{FM}} E_{0}^{1 / 6} M_{00} t$, and $(5 \sqrt{2} / 6) a_{0}\left(\Omega_{\infty}\right) b_{0}\left(\Omega_{\infty}\right)=2.580$. The time evolution of the dimensionless zeroth moment for other methods also has the same form:

$$
\left.\frac{M_{0}}{M_{00}}\right|_{\mathrm{SPSD}}=(\gamma \tau+1)^{-6 / 5},
$$

where $\gamma=(5 \sqrt{2} / 6) a_{0}\left(\Omega_{\infty}\right) b_{0}\left(\Omega_{\infty}\right)$, the value of $\gamma$ is 2.604 for the $\log$ MOM (Lee et al. 1984), 2.779 for the STM (Lai et al. 1972), and 2.630 for the TEMOM (Chen et al. 2014). Figure 4a illustrates the evolution of the dimensionless zeroth moment produced by different methods for Brownian coagulation in the free-molecular regime. The initial condition for the investigated aerosol is assumed to be already under the SPSD state. The analytical solutions from the present IGDMOM, TEMOM, and $\log$ MOM had good agreement for the evolution of $M_{0} / M_{00}$. However, obvious differences existed between the STM (Lai et al. 1972) and these three MOMs, namely, the IGDMOM, TEMOM, and $\log$ MOM.

The time evolution of the dimensionless second moment $M_{2} M_{00} / M_{10}^{2}$ can be derived using Eq. (20), if the initial value $\Omega_{0}$ is evaluated as $\Omega_{\infty}$, the shape factor of $\Omega$ for the SPSD in the free-molecular regime is

$$
\left.\frac{M_{2} M_{00}}{M_{10}^{2}}\right|_{\text {SPSD }}=\Omega_{\infty}\left[\frac{5 \sqrt{2}}{6} a_{0}\left(\Omega_{\infty}\right) b_{0}\left(\Omega_{\infty}\right) \tau+1\right]^{6 / 5} .
$$

Accordingly, a unified expression can be proposed for all the existing methods, including the present IGDMOM, log MOM, TEMOM, and STM:

$$
\left.\frac{M_{2}}{M_{20}}\right|_{\text {SPSD }}=(\gamma \tau+1)^{6 / 5} .
$$

Figure $4 \mathrm{~b}$ illustrates the evolution of the dimensionless second moment predicted using different methods. The present IGDMOM, TEMOM, and log MOM produce overlapped curves, which cannot be distinguished from each other.

In conclusion, we observed that for dimensionless moments, namely, $M_{0} / M_{00}$ and $M_{2} / M_{20}$, unified expressions exist in all existing methods. There are only differences for specific parameters among all the methods. The analytical expressions derived using the IGDMOM provide great precision.

c. SPT

According to the SPSD theory by Friedlander (1960), a coagulating aerosol in an atmospheric environment should reach 

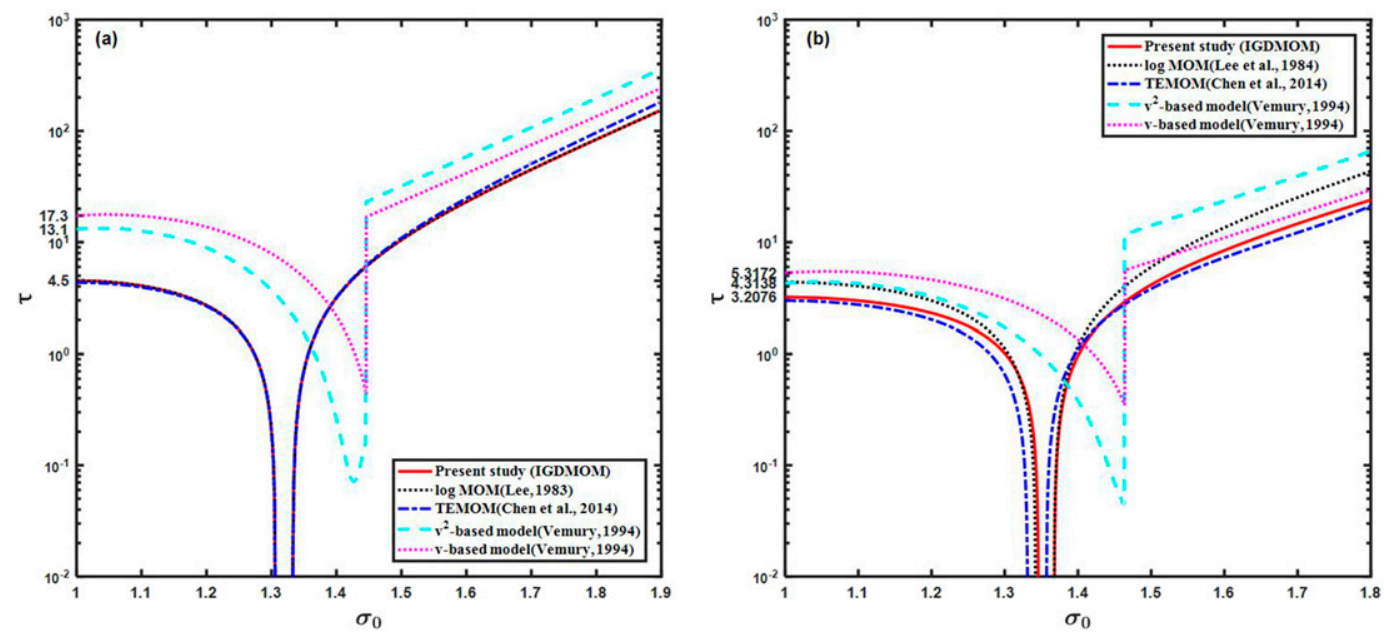

FIG. 5. Time to attain the self-preserving distribution in the (a) continuum regime and (b) free-molecular regime as a function of the initial geometric standard deviation for various methods.

the same steady-state size distribution with time regardless of the aerosol's initial size distribution. When this steady state is reached, the shape of aerosol size distribution dealt by similarity transformation treatment does not change with time (Friedlander 1960, 1961; Friedlander and Wang 1966; Wang and Friedlander 1967). Clark and Whitby (1967) used the theory to successfully explain the general shape of the observed size distribution of atmospheric aerosols. Although in the atmospheric environment, the aerosol size distributions in any position may be different, the size distribution can be locally SPSD at all times. Thus, for predicting the property of atmospheric aerosol at a given time, determining the time required to attain the SPSD; that is, SPT is essential.

In this study, the SPTs calculated from the present model presented in Eqs. (35) and (37) are compared with those from the log MOM and TEMOM. The SMs ( $v^{2}$-based model, $f_{s}=$ 1.08; $v$-based model, $f_{s}=1.08$ ) used by Vemury et al. (1994) were executed as a reference, whose detailed mathematical expression is presented in appendix F. Figure 5a illustrates the SPT in the continuum regime as a function of the initial geometric standard deviation $\sigma_{g 0}$. The selection of $\sigma_{g 0}$ rather than the shape factor $\Omega_{0}$ as abscissa was consistent with (Vemury et al. 1994). Here, following the definition for $\sigma_{g}$ in the $\log$ MOM leads to the relation between $\sigma_{g 0}$ and $\Omega_{0}$ (Pratsinis 1988):

$$
\ln \sigma_{g 0}=\frac{1}{3} \sqrt{\ln \Omega_{0}}
$$

Although the MOMs, including the IGDMOM, TEMOM, and $\log$ MOM, generate nearly overlapping results for all $\sigma_{g 0}$, substantial differences exist between the MOMs and the SMs $\left(v^{2}\right.$-and $v$-based models). The obvious difference between the log MOM and SMs was addressed by Vemury et al. (1994). Figure $5 \mathrm{~b}$ illustrates the SPT in the free-molecular regime as a function of the initial geometric standard deviation $\sigma_{g 0}$.
Notably, at the SPSD state near $\sigma_{g 0} \approx 1.345$, the present model overlaps with the $\log$ MOM, whereas away from $\sigma_{g 0} \approx 1.345$, the present study approaches the TEMOM. Similar to the discussion in Fig. 5a, all three MOMs deviate substantially from the SMs ( $v^{2}$ - and $v$-based models). In Table 1 , the SPTs are presented for the various methods when $\Omega_{0}=1$. For comparison, the $v^{2}$-based model, $v$-based model, log MOM, and TEMOM are selected as references. The present IGDMOM yields the same SPT for Brownian coagulation in the continuum regime as $\log$ MOM, and the value lies between that obtained using the log MOM and TEMOM for Brownian coagulation in the free-molecular regime.

Figure 5 illustrates that the further the value of $\sigma_{g 0}$ is from the value in the SPSD at time $\tau=0$, the longer it takes for the aerosol to reach the SPSD. This finding is quantitatively in agreement with Hidy (1965) in that the self-preserving spectrum is independent of $n(v, 0)$ although the time required to reach the SPSD depends on the shape of the initial distribution.

\section{d. SPSD}

The SPSD theory has become a critical tool for exploring atmospheric particle coagulation mechanisms (Kreidenweis et al. 2019; Pruppacher and Klett 1997). Friedlander and Wang (1966) studied the theoretical coagulation equation in the

TABLE 1 . SPT when $\Omega_{0}=1$. For $v^{2}$-based and $v$-based models $f_{s}=1.08$.

\begin{tabular}{lcc}
\hline \multicolumn{1}{c}{ Methods } & $\begin{array}{c}\text { Continuum } \\
\text { regime }\end{array}$ & $\begin{array}{c}\text { Free-molecular } \\
\text { regime }\end{array}$ \\
\hline $\log$ MOM (Lee 1983) & 4.5 & $/$ \\
log MOM (Lee et al. 1984) & - & 4.3797 \\
TEMOM (Chen et al. 2014) & 4.3520 & 2.9868 \\
$v^{2}$-based model (Vemury et al. 1994) & 13.1 & 4.3 \\
$v$-based model (Vemury et al. 1994) & 17.3 & 5.3 \\
IGDMOM (present study) & 4.5 & 3.2076 \\
\hline
\end{tabular}



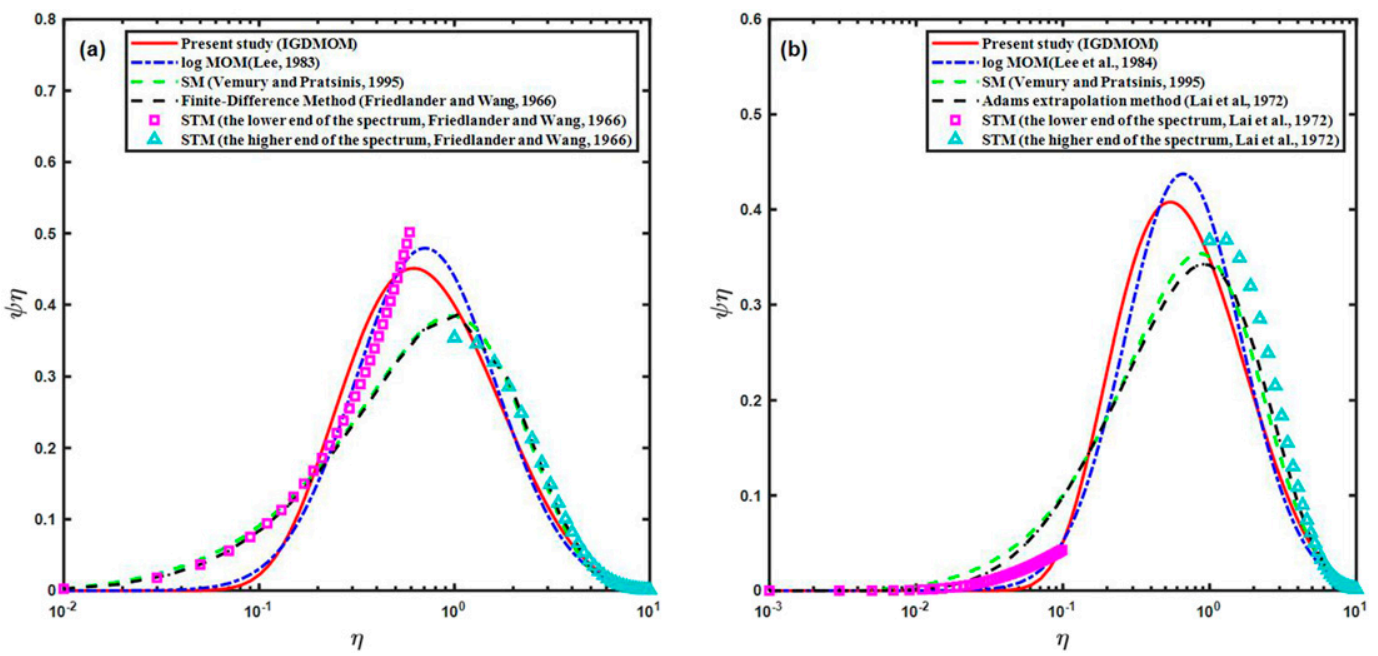

FIG. 6. Self-preserving size distribution (SPSD) for different methods in the (a) continuum regime and (b) freemolecular regime.

continuum regime by using the STM, and Lai et al. (1972) used the same similarity solution to study the coagulation equation in the free-molecular regime. On the basis of the assumption that the PSD is lognormal distribution, through using the transformation formula in STM [i.e., Eq. (28)], Lee (1983) and Lee et al. (1984) derived new analytical SPSD expressions for atmospheric aerosols underlying Brownian coagulation in both continuum and free-molecular regimes. Such solutions by Friedlander and Lee have become the main tools for determining atmospheric aerosol asymptotic distribution after long periods.

Therefore, comparing the SPSD obtained from the IGDMOM with solutions obtained from the STM by Friedlander and log MOM by Lee is essential, and such a comparison is presented in Fig. 6. In Fig. 6a, two direct numerical solutions to the KCE regarding Brownian coagulation in the continuum regime are displayed as a reference, namely, the finite-difference method used by Friedlander and Wang (1966) and the SM used by Vemury and Pratsinis (1994). These two numerical methods nearly overlap each other, although different numerical techniques are used. Both the log MOM and IGDMOM provide a continuous spectrum over the whole size distribution, whereas the STM by Friedlander and Wang provides only reasonable spectra in the lower and higher ends of size distributions. Friedlander and Wang (1966) claimed that the STM agrees well only with the numerical solutions for $\eta<0.1$ and $\eta>0.1$, which are also presented in Fig. 6a. Comparing the IGDMOM with the $\log$ MOM and STM, the IGDMOM is much closer to the $\log$ MOM over the whole size distribution, although these two methods seem different from the referenced numerical solutions.

Figure 6b compares the present IGDMOM, log MOM, and STM for the SPSD regarding Brownian coagulation in the freemolecular regime. For reference, we adopted the SM used by Vemury and Pratsinis (1994) and the Adams extrapolation method used by Lai et al. (1972). SPSD expressions in the log MOM and the STM are proposed by Lee et al. (1984) and Lai et al. (1972). The STM in Fig. 6b gives only the fitted values to the numerical solutions in the upper and lower ends of the size distribution, but the fitting is not as good as the STM in Fig. 6a. Figure $6 \mathrm{~b}$ presents that the satisfying agreement between the STM and numerical solutions is only for $\eta<0.01$ and $\eta>5.0$. Similar to the presentation in Fig. 6a, the IGDMOM is much closer to the log MOM than to other solutions.

As is illustrated in Figs. 6a and 6b, the STM can only provide the spectra in the upper and lower ends of the size distribution, although these two little ends fit the referenced solution given by the SM well. The present IGDMOM straightly gives the analytical and explicit expression for the whole size distribution. The difference between the IGDMOM and the referenced solution is due to the assumption of the time dependent distribution.

\section{Conclusions}

A scheme to realize the SPSD theory is presented in this study. The scheme is executed by first introducing IGDMOM into the description of the atmospheric aerosol size distribution and then by analytically solving the KCE in terms of realistic Brownian coagulation kernels in the continuum and free-molecular regimes. The KCE is solved using the concept of the MOM in which the governing equations for the first three moments are needed. We demonstrate the ability of the new scheme for realizing the SPSD theory by presenting new reasonable mathematical expressions for the SPSD and SPT.

The present scheme is mainly compared with other several recognizable schemes, including the SM, STM, log MOM, and TEMOM. Regarding analytical solutions to the KCE, the SPT, and SPSD expression, our new scheme is verified to realize the SPSD theory with nearly the same accuracy as or higher accuracy than the log MOM, and shows obvious higher accuracy than the schemes by Smoluchowski (1917) and Friedlander and Wang (1966). The scheme presented in this study demonstrates 
the SPSD theory more reasonably than the other schemes, such as the STM and $\log$ MOM, since the derivations for expressions of the SPSD and SPT are only based on rigorous theoretical analysis.

The SPSD expressions from the present study are straightforward and explicit functions for the whole spectra. Previously, analytical solutions could only be obtained from extreme ranges. To obtain a full range of solutions through STM (Friedlander and Wang 1966), the integrodifferential equations were handled using numerical methods, with several key coefficients obtained from other numerical solutions (Friedlander and Wang 1966; Lai et al. 1972).

The present scheme provides additional proof for the SPSD theory and has important implications for developing tropospheric atmospheric aerosol dynamics models involving the evolution of size distribution.

Acknowledgments. The authors thank the National Natural Science Foundation of China (11872353, 91852102) and the Zhejiang Provincial Natural Science Foundation of China (LR16A020002) for their support. Additionally, we are grateful to the anonymous reviewers for their suggestions.

\section{APPENDIX A}

\section{Method for Obtaining $b_{0}$ and $b_{2}$ Presented in Eq. (11)}

In the IGDMOM, we approximated $\left(1 / v+1 / v_{1}\right)^{1 / 2}$ with $\sqrt{2} b_{i} v^{-1 / 4} v_{1}^{-1 / 4}, i=0,2$, where $b_{i}$ was computed as follows:

$$
b_{i}=\frac{\int_{0}^{\infty} \int_{0}^{\infty}\left(v v_{1}\right)^{k / 2}\left(\frac{1}{v}+\frac{1}{v_{1}}\right)^{1 / 2}\left(v^{1 / 3}+v_{1}^{1 / 3}\right)^{2} n(v) n\left(v_{1}\right) d v d v_{1}}{\int_{0}^{\infty} \int_{0}^{\infty}\left(v v_{1}\right)^{k / 2} \sqrt{2} v^{-1 / 4} v_{1}^{-1 / 4}\left(v^{1 / 3}+v_{1}^{1 / 3}\right)^{2} n(v) n\left(v_{1}\right) d v d v_{1}}
$$

Notably, the values of $b_{0}$ and $b_{2}$ change only with the ratio of $\varphi$ and $\phi$ (i.e., $\varphi / \phi=s$ ), rather than their absolute magnitude. The different values of $b_{0}$ and $b_{2}$ following the variance of $s$ are indicated in Shen et al. (2020b), and we fit $b_{0}$ and $b_{2}$ in line with $s$ from 0.1 to 1.1 with the interval of 0.1 , as follows:

$$
b_{0}(s)=0.1658 s^{-0.4754}+0.9267
$$

and

$$
b_{2}(s)=0.226 s^{-0.3378}+0.8643 \text {. }
$$

Here, the solution for $b_{i}$ differs from that by Lee et al. in that the $\log$ MOM has lower accuracy than the IGDMOM (Lee et al. 1984; Shen et al. 2020b).

\section{APPENDIX B}

\section{Analytical Solutions to the KCE by Various Scientists}

Smoluchowski (1917) was the first to provide an analytical expression for an early coagulation stage of monodisperse aerosols. Lee (1983) presents the classical analytical solution to the KCE with Smoluchowski's monodisperse assumption:

$$
\frac{M_{0}}{M_{00}}=\frac{1}{1+2 \tau},
$$

where $\tau=K_{\mathrm{CO}} M_{00} t, M_{00}$ is the initial value for $M_{0}$ at $t=0$. Because of the assumption of a constant collision kernel, Smoluchowski's solution is expected to apply only to the early stages of coagulation; however, this solution has reasonably good agreement with numerical calculations for predicting the number decay even for late stages. Equation (B1) was applied by Turco and Yu (1998) to the analytical study of aerosol size distribution in a coagulating plume.

To overcome the limit of Smoluchowski's scheme, which only applies to monodisperse particles, Friedlander et al. proposed using the similarity transformation to obtain the solution to the KCE once the PSD becomes the SPSD (Wang and Friendlander 1967; Friedlander 1960, 1961). In the continuum regime, the decay of number concentrations takes the following expression (Lee 1983):

$$
\frac{M_{0}}{M_{00}}=\frac{1}{1+(1+a b) \tau},
$$

where $a=0.9046$ and $b=1.248$. In the free-molecular regime, the corresponding decay of number concentrations using Friedlander's STM takes the following expression:

$$
\frac{M_{0}}{M_{00}}=(c \tau+1)^{-6 / 5},
$$

where $c=(5 / 6)(\alpha / 2)=2.7791, \tau=K_{\mathrm{FM}} E_{0}^{1 / 6} M_{00} t, M_{00}$, and $E_{0}$ are total particle number concentration and mean particle volume of an aerosol at the time, $t=0$. In this study, $E_{0}$ is $\phi$ at $t=0$.

Friedlander's work could not provide the time evolution of PSDs before the SPSD state was achieved (Friedlander 1960, 1961). To quantitatively examine many noteworthy timedependent characteristics of the Brownian coagulation process, such as the effects of time and the initial spread of the PSD on the number decay, on the increase of the mean particle size, and on the change in the standard deviation, for the first time, Lee (1983) used MOMs to obtain a simple analytical solution that provides the size distribution over the entire period of coagulation, based on the use of a time-dependent lognormal function for depicting the size distribution of coagulating particles (Lee 1983; Lee et al. 1984). In the continuum regime, Lee (1983) obtained the analytical solution to the $\mathrm{KCE}$ :

$$
\left\{\begin{array}{c}
\frac{M_{0}}{M_{00}}=\frac{1}{\left(1+\Omega_{0}^{1 / 9}\right) \tau+1}, \\
\frac{M_{2}}{M_{20}}=\frac{2}{\Omega_{0}}\left(1+\Omega_{0}^{1 / 9}\right) \tau+1,
\end{array}\right.
$$

where $\tau=K_{\mathrm{CO}} M_{00} t$, and $\Omega_{0}$ is the value at the initial time.

In the free-molecular regime, Lee's analytical solution (Lee et al. 1984) to the KCE takes the form of 


$$
\left\{\begin{array}{c}
\frac{M_{0}}{M_{00}}=\left[\frac{5}{6}\left(\Omega_{0}^{-5 / 72}+2 \Omega_{0}^{-1 / 72}+\Omega_{0}^{19 / 72}\right) b \tau+1\right]^{-6 / 5}, \\
\frac{M_{2}}{M_{20}}=\left[\frac{5}{3}\left(\Omega_{0}^{-5 / 72}+2 \Omega_{0}^{-1 / 72}+\Omega_{0}^{19 / 72}\right) \Omega_{0}^{-5 / 6} \frac{5}{6} b \tau+1\right]^{6 / 5},
\end{array}\right.
$$

where $\tau=K_{\mathrm{FM}} E_{0}^{1 / 6} M_{00} t$, and $b$ is a constant.

\section{APPENDIX C}

\section{Derivation of Eq. (14) for Different Methods}

\section{a. IGDMOM}

The $k$ th moment closure function of the IGDMOM is presented in Eq. (3), which yields

$$
\begin{aligned}
M_{k} & =M_{0} E^{k} \sqrt{\frac{2}{\pi}} s^{1 / 2} e^{s} K_{k-1 / 2}(s) \\
& =M_{0} E^{k} f(\Omega, k),
\end{aligned}
$$

where $s=1 /(\Omega-1)$.

\section{b. $\log M O M$}

The $k$ th moment closure function of $\log$ MOM was first given by Lee (1983):

$$
\begin{aligned}
M_{k} & =M_{0} v_{g}^{k} e^{(9 / 2) k^{2} \ln ^{2} \sigma} \\
& =M_{0} v_{g}^{k}\left(e^{9 \ln ^{2} \sigma}\right)^{k^{2} / 2} \\
& =M_{0}\left(\frac{M_{1}}{M_{0}}\right)^{k}\left(\frac{1}{\Omega}\right)^{k / 2}(\Omega)^{k^{2} / 2} \\
& =M_{0} E^{k} \Omega^{\left(k^{2}-k\right) / 2} \\
& =M_{0} E^{k} f(\Omega, k),
\end{aligned}
$$

where $v_{g}=\left(M_{1} / M_{0}\right)\left[M_{1}^{2} /\left(M_{0} M_{2}\right)\right]^{1 / 2}$, and $\Omega=e^{9 \ln ^{2} \sigma}$.

\section{c. TEMOM}

The $k$ th moment closure function of the TEMOM was observed by Yu et al. (2008):

$$
\begin{aligned}
M_{k} & =\frac{u^{k-2}}{2}\left(k^{2}-k\right) M_{2}+u^{k-1}\left(-k^{2}+2 k\right) M_{1}+u^{k}\left(\frac{k^{2}}{2}-\frac{3}{2} k+1\right) M_{0} \\
& =\frac{\left(k^{2}-k\right)}{2}\left(\frac{M_{1}}{M_{0}}\right)^{k-2} M_{2}+\left(-k^{2}+2 k\right)\left(\frac{M_{1}}{M_{0}}\right)^{k-1} M_{1}+\left(\frac{k^{2}}{2}-\frac{3}{2} k+1\right)\left(\frac{M_{1}}{M_{0}}\right)^{k} M_{0} \\
& =M_{0} E^{k}\left[\frac{k^{2}-k}{2} \Omega-\frac{k^{2}-k}{2}+1\right] \\
& =M_{0} E^{k} f(\Omega, k) .
\end{aligned}
$$

\section{APPENDIX D}

\section{Derivation of Eq. (15)}

In mathematics, a function $a(s)$ exists, which makes the following equality holds for $s$ :

$$
K_{1 / 6}(s) K_{5 / 6}(s)=a(s)\left[K_{1 / 2}(s)\right]^{2},
$$

where $a(s)$ is a monotonically decreasing function for $s>0$ and with the following constraint:

$$
\begin{gathered}
\lim _{s \rightarrow 0} a(s)=2, \\
\lim _{s \rightarrow+\infty} a(s)=1, \\
\frac{d}{d s} a(s)<0 .
\end{gathered}
$$

The variance of $a(s)$ is very small in the interval $(0,+\infty)$, and it has a high convergence rate to 1 . When $s=0.5$, the value of $a(0.5)$ is equal to 1.1388 . Some values of $a(s)$ with $s$ are presented in Table D1, and the curve displaying the variance of $a(s)$ with $s$ is illustrated in Fig. D1.

Combining with Eq. (D1), Eq. (10) can be rewritten as

$$
\left\{\begin{array}{c}
\frac{d M_{0}}{d t}=-(1+a(s)) K_{\mathrm{CO}} M_{0}^{2} \\
\frac{d M_{1}}{d t}=0 \\
\frac{d M_{2}=}{d t}=2[1+a(s)] K_{\mathrm{CO}} M_{1}^{2}
\end{array}\right.
$$

The parameter $a(s)$ can be regarded as a constant when solving Eq. (D2) because it does not diverge but always converges quickly to a certain value during coagulation, and the difference between the maximum and the minimum is negligible. To facilitate the calculation, we choose the initial value $a\left(s_{0}\right)$. Next, Eq. (15) can be obtained from Eq. (D2). 
TABLE D1. Values of $a$ for $s$.

\begin{tabular}{cc}
\hline \hline$s$ & $a(s)$ \\
\hline 0.5 & 1.133851 \\
0.6 & 1.118271 \\
0.7 & 1.106102 \\
0.8 & 1.096304 \\
0.9 & 1.088226 \\
1.0 & 1.081442 \\
1.1 & 1.075657 \\
1.2 & 1.070660 \\
1.3 & 1.066299 \\
1.4 & 1.062456 \\
1.5 & 1.059044 \\
$\infty$ & 1 \\
\hline
\end{tabular}

\section{APPENDIX E}

\section{Derivation of Eq. (20)}

We define two functions $a_{0}(s)$ and $a_{2}(s)$ to satisfy the following equality:

$$
K_{1 / 12}(s) K_{3 / 4}(s)+\left[K_{5 / 12}(s)\right]^{2}=a_{0}(s)\left[K_{1 / 2}(s)\right]^{2}
$$

and

$$
K_{11 / 12}(s) K_{1 / 4}(s)+\left[K_{7 / 12}(s)\right]^{2}=a_{2}(s)\left[K_{1 / 2}(s)\right]^{2},
$$

for $s$ in which $a_{0}(s)$ and $a_{2}(s)$ are monotonically increasing and decreasing functions for $s>0$, respectively, with the constraints that

$$
\begin{aligned}
& \lim _{s \rightarrow 0} a_{0}(s)=0, \quad \lim _{s \rightarrow+\infty} a_{0}(s)=2, \quad \frac{d}{d s} a_{0}(s)>0 ; \\
& \lim _{s \rightarrow 0} a_{2}(s)=+\infty, \quad \lim _{s \rightarrow+\infty} a_{2}(s)=2, \quad \frac{d}{d s} a_{2}(s)<0 .
\end{aligned}
$$

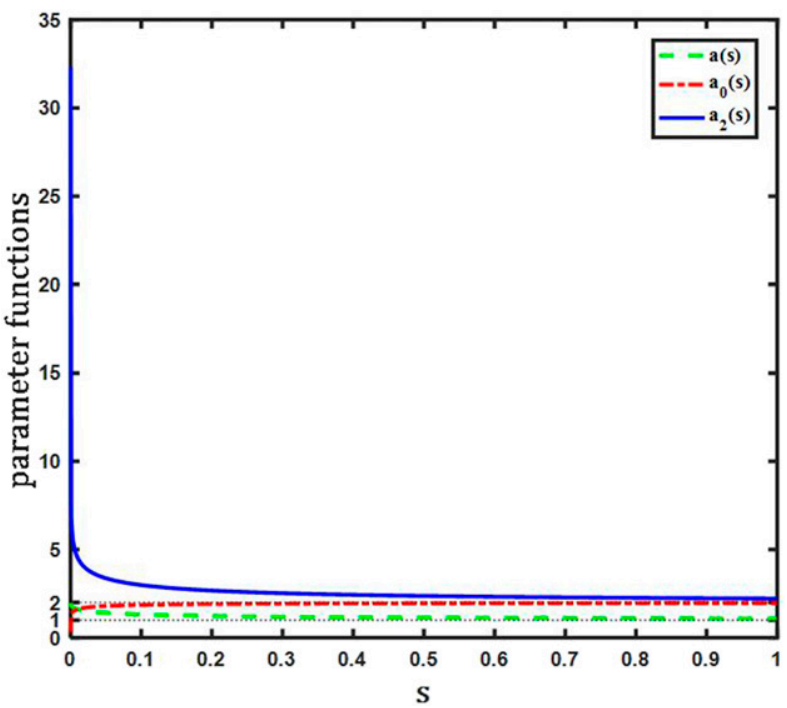

FIG. D1. Three parameter functions in the analytical solutions.
TABLE E1. Values of $a_{0}, a_{2}, b_{0}$, and $b_{2}$ for $s$.

\begin{tabular}{lcccc}
\hline \multicolumn{1}{c}{$s$} & $a_{0}$ & $a_{2}$ & $b_{0}$ & $b_{2}$ \\
\hline 0.1 & 1.861928 & 2.988801 & 1.421529 & 1.35528 \\
0.2 & 1.908277 & 2.674563 & 1.284650 & 1.255496 \\
0.3 & 1.929314 & 2.524918 & 1.220996 & 1.204311 \\
0.4 & 1.941801 & 2.433792 & 1.182569 & 1.171774 \\
0.5 & 1.95022 & 2.371422 & 1.156369 & 1.14885 \\
0.6 & 1.956344 & 2.325642 & 1.137172 & 1.131666 \\
0.7603 & 1.963364 & 2.272804 & 1.115030 & 1.111441 \\
0.8 & 1.964743 & 2.262382 & 1.110668 & 1.107400 \\
0.9 & 1.967772 & 2.239479 & 1.101073 & 1.098461 \\
1.0 & 1.970294 & 2.220387 & 1.093072 & 1.090946 \\
1.1 & 1.972431 & 2.204206 & 1.086288 & 1.084531 \\
$\infty$ & 2 & 2 & 1 & 1 \\
\hline
\end{tabular}

The properties of $a_{0}(s)$ imply that the variance with $s$ is very small in the interval $(0,+\infty)$, and it has a high convergence rate to 2 [e.g., $a_{0}(0.5)=1.9502$ ], and notably, $a_{2}(\mathrm{~s})$ also has a high convergence rate to 2 [e.g., $a_{2}(0.5)=2.3714$ ]. Some values of $a_{0}(s)$ and $a_{2}(s)$ with $s$ are presented in Table E1, and the curves displaying the variance of them with $s$ are illustrated in Fig. D1.

According to the above clarification, Eq. (11) can be rewritten as

$$
\begin{aligned}
\frac{d M_{0}}{d t} & =-\sqrt{2} K_{\mathrm{FM}} b_{0}(s) M_{1}^{2} \phi^{-11 / 6} a_{0}(s), \\
\frac{d M_{1}}{d t} & =0, \\
\frac{d M_{2}}{d t} & =2 \sqrt{2} K_{\mathrm{FM}} b_{2}(s) M_{1}^{2} \phi^{1 / 6} a_{2}(s) .
\end{aligned}
$$

On the basis of the characteristics of $a_{k}(s)$ and $b_{k}(s)(k=1$, 2), they can be considered as constants; accordingly, Eq. (E3a) yields

$$
\frac{M_{0}}{M_{00}}=\left[\frac{5 \sqrt{2}}{6} a_{0}(s) b_{0}(s) \tau+1\right]^{-6 / 5},
$$

where $M_{00}$ is the initial value of $M_{0}$, and $\tau=K_{\mathrm{FM}} E_{0}^{1 / 6} M_{00} t$. Following Xie and He's solution to divide Eq. (E3a) by Eq. (E3b) (Xie and He 2013), the following equation is derived:

$$
\frac{d M_{0}}{d M_{2}}=-\frac{a_{0}(s) b_{0}(s)}{2 a_{2}(s) b_{2}(s)} \frac{M_{0}^{2}}{M_{1}^{2}} .
$$

Let $\left[a_{0}(s) b_{0}(s)\right] /\left[2 a_{2}(s) b_{2}(s)\right]=A$, and considering that the calculation formula in Eq. (A1) additionally indicates that $b_{0}$ and $b_{2}$ are almost always identical, $A$ can be redefined as $A=a_{0}(s) /\left[2 a_{2}(s)\right]$. Solving Eq. (E5) and combining with Eq. (E4) leads to the analytical solution of $M_{2}$ :

$$
\frac{M_{2}}{M_{20}}=\frac{1}{A \Omega_{0}}\left\{\left[\frac{5 \sqrt{2}}{6} a_{0}(s) b_{0}(s) \tau+1\right]^{6 / 5}+A \Omega_{0}-1\right\},
$$

where $M_{20}$ is the initial value of $M_{2}$. 


\section{APPENDIX F}

\section{SPT of $\boldsymbol{v}^{\mathbf{2}}$-Based Model and $\boldsymbol{v}$-Based Model}

For $v^{2}$ - and $v$-based models (Vemury et al. 1994), the SPT in the continuum regime is

$$
\tau_{\infty}=\left\{\begin{array}{cc}
A_{c} \sigma_{g 0}-B_{c}\left(\sigma_{g 0}-1\right)^{2} \exp \left[-C_{c}\left(\sigma_{g 0}-1\right)^{2}\right], & \sigma_{g 0}<\sigma_{\mathrm{gsc}} \\
10^{D_{c}+E_{c} \sigma_{g 0}}, & \sigma_{g 0}>\sigma_{\mathrm{gsc}}
\end{array}\right.
$$

For the $v^{2}$-based model, $\sigma_{\mathrm{gsc}}=1.445, A_{c}=13.1, B_{c}=180.3$, $C_{c}=7.3, D_{c}=-2.44$, and $E_{c}=2.63$, whereas for the $v$-based model, $\sigma_{\mathrm{gsc}}=1.446, A_{c}=17.3, B_{c}=182.6, C_{c}=4.4$, $D_{c}=-2.46$, and $E_{c}=2.55$.

For $v^{2}$ - and $v$-based models in the free-molecular regime (Vemury et al. 1994), the SPT is

$$
\tau_{\infty}=\left\{\begin{array}{cc}
A_{f} \sigma_{g 0}-B_{f}\left(\sigma_{g 0}-1\right)^{2} \exp \left[-C_{f}\left(\sigma_{g 0}-1\right)^{2}\right], & \sigma_{g 0}<\sigma_{\mathrm{gsf}} \\
10^{D_{f}+E_{f} \sigma_{g 0}}, & \sigma_{g 0}>\sigma_{\mathrm{gsf}}
\end{array} .\right.
$$

For the $v^{2}$-based model, $\sigma_{\mathrm{gsf}}=1.463, A_{f}=4.3, B_{f}=49.8, C_{f}=$ 5.4, $D_{f}=-2.18$, and $E_{f}=2.22$, and for the $v$-based model, $\sigma_{\mathrm{gsf}}=1.464, A_{f}=5.3, B_{f}=45.1, C_{f}=2.7, D_{f}=-2.4$, and $E_{f}=2.15$.

\section{APPENDIX G}

\section{The Case of SPSD in the Condensation}

The description of condensation can be referenced in Shen et al. (2020b). Here, it will be discussed in more detail, including its numerical and analytical solution.

Unlike coagulation, condensation is the process by which gas molecules are deposited on the surface of existing particles, resulting in particle growth but not affecting particle number (Li et al. 2020). The condensation rate has the following universal mathematical form (Yu et al. 2017):

$$
G=\vartheta v^{x},
$$

where $\vartheta$ and $x$ are constant with time, which depends on the saturation ratio and physical parameters of coating molecules. Here, let $\vartheta=1$ without loss of generality and $x=1 / 3$ for spherical particles. By substituting Eq. (G1) into

$$
\left.\frac{\partial n(v, t)}{\partial t}\right|_{\mathrm{CON}}=\frac{\partial G n(v, t)}{\partial v},
$$

where $n(v, t)$ is the particle number density for particle volume $v$ and time $t, G$ is the condensation growth rate, we obtain the moment ODEs by using the universal formula of condensation:

$$
\left.\frac{d M_{\mathrm{k}}}{d t}\right|_{\mathrm{CON}}=k \vartheta M_{k+x-1}(k=0,1,2, \ldots) .
$$

This is the universal moment ODE for condensation, regardless of the size regime.
By introducing Eq. (3) into Eq. (G3), the ODEs with respect to moments are obtained:

$$
\left\{\begin{array}{c}
\left.\frac{d M_{0}}{d t}\right|_{\mathrm{CON}}=0, \\
\left.\frac{d M_{1}}{d t}\right|_{\mathrm{CON}}=\vartheta M_{1} \sqrt{\frac{2}{\pi}} s^{1 / 2} e^{s} \psi^{x-1} K_{x-1 / 2}(s), \\
\left.\frac{d M_{2}}{d t}\right|_{\mathrm{CON}}=2 \vartheta M_{1} \sqrt{\frac{2}{\pi}} s^{1 / 2} e^{s} \psi^{x} K_{x+1 / 2}(s) .
\end{array}\right.
$$

With $\sqrt{2 / \pi} s^{1 / 2} e^{s}=1 / K_{1 / 2}(s)$, Eq. (G4) then can be rewritten as

$$
\left\{\begin{array}{c}
\left.\frac{d M_{0}}{d t}\right|_{\mathrm{CON}}=0 \\
\left.\frac{d M_{1}}{d t}\right|_{\mathrm{CON}}=\vartheta M_{1} \psi^{-2 / 3} \frac{K_{-1 / 6}(s)}{K_{1 / 2}(s)} \\
\left.\frac{d M_{2}}{d t}\right|_{\mathrm{CON}}=2 \vartheta M_{1} \psi^{1 / 3} \frac{K_{5 / 6}(s)}{K_{1 / 2}(s)}
\end{array}\right.
$$

Then the definition of the shape factor $\Omega=M_{0} M_{2} / M_{1}^{2}$ gives its derivative:

$$
\left.\frac{d \Omega}{d t}\right|_{\mathrm{CON}}=2 \vartheta\left(\frac{M_{0}}{M_{1}}\right)^{2 / 3}\left[\frac{K_{5 / 6}(s)}{K_{1 / 2}(s)}-\Omega \frac{K_{1 / 6}(s)}{K_{1 / 2}(s)}\right] .
$$

Following the characteristics of the second type of modified Bessel function, the assumption $K_{5 / 6}(s) / K_{1 / 2}(s)=A$ and $K_{1 / 6}(s) / K_{1 / 2}(s)=B$ can be adopted. Then Eq. (G6) is rewritten as

$$
\left.\frac{d \Omega}{d t}\right|_{\mathrm{CON}}=2 \vartheta\left(\frac{M_{0}}{M_{1}}\right)^{2 / 3}(A-B \Omega) .
$$

Combining the second equation in Eq. (G5) and Eq. (G7) yields

$$
\left.\frac{d \Omega}{d M_{1}}\right|_{\mathrm{CON}}=\frac{2}{M_{1}}[] \frac{K_{5 / 6}(s)}{K_{1 / 6}(s)}-\Omega .
$$

For the difficulty caused by $K_{5 / 6}(s) / K_{1 / 6}(s)=C$, its initial value is chosen, then

$$
M_{1}^{2}=\frac{\left(\Omega_{0}-C_{0}\right) M_{10}^{2}}{\Omega-C} .
$$

Substituting Eq. (G9) into Eq. (G7) gives the formula of $\Omega$ with respect to time,

$$
\begin{aligned}
& t-\frac{\ln (\alpha+\eta)}{D B \eta}+\frac{\ln \left(\alpha^{2}-\alpha \eta+\eta^{2}\right)}{2 D B \eta} \\
& +\frac{\sqrt{3} \arctan \left[\frac{\sqrt{3}}{3}\left(\frac{2 \alpha}{\eta}-1\right)\right]}{D B \eta}+\text { constant }=0,
\end{aligned}
$$

where $D=2\left(M_{0} / M_{10}\right)^{2 / 3}\left[1 /\left(\Omega_{0}-C_{0}\right)\right]^{1 / 3}, \alpha=(\Omega-C)^{1 / 3}, \eta=$ $[(B C-A) / B]^{1 / 3}$, and the value of constant is calculated when $t=0$. Thus, the analytical solutions of $\Omega$ and $M_{1}$ can be obtained from Eqs. (G10) and (G9). They are compared with the 

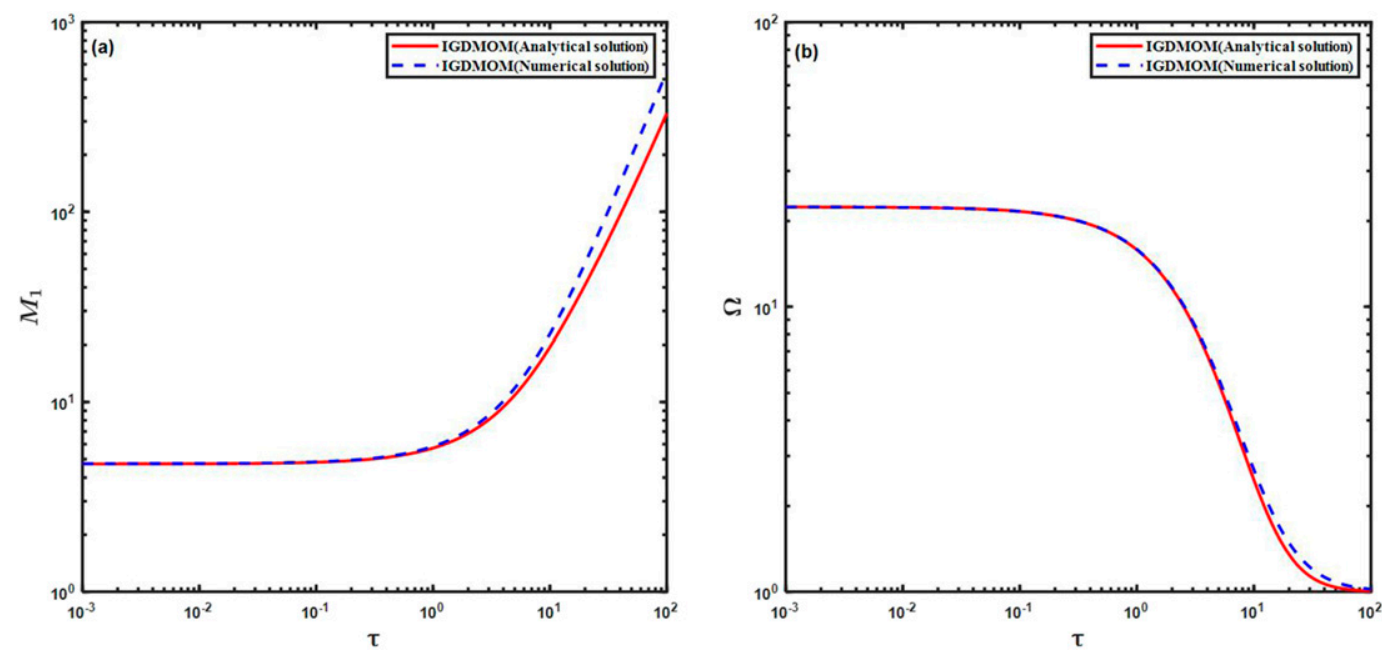

FIG. G1. Variances of (a) the first moment and (b) the shape factor with time in the condensation.

numerical solutions from Eq. (G5), and the results are presented in Fig. G1. By computation of Eq. (G10), the limit of $\Omega$ is 1 , that is, the limit of $s=1 /(\Omega-1)$ is $+\infty$, implying that there is no limit for the parameter $s$ in the PSD of the IGD, which causes there to be no SPSD for the condensation because of the function $\psi(\eta)$ in Eq. (31) is 0 when $s_{\infty}$ is $+\infty$. This conclusion coincides with the real-world situation, which also verifies the effectiveness of the IGDMOM.

\section{APPENDIX H}

\section{Nomenclature, Greek Letters, and Abbreviations}

\section{a. Nomenclature}

$v \quad$ Particle volume

$N \quad$ Particle number concentration density

$M_{k} \quad k$ th moment of particle size distribution

$N(t) \quad$ Total particle number concentration at time $t$

$N_{0} \quad$ Initial total particle number concentration

$C$ Cunningham correction factor

$k_{B} \quad$ Boltzmann constant, J·K

Kn Particle Knudsen number

$t \quad$ Time, $\mathrm{s}$

$T$ Temperature, $\mathrm{K}$

$r \quad$ Aerosol particle radius

$d_{p 0} \quad$ Initial mean diameter of aerosol

\section{b. Greek letters}

$\nu \quad$ Kinematic viscosity, $\mathrm{m}^{2} \mathrm{~s}^{-1}$

$\beta \quad$ Particle collision kernel

$\mu \quad$ Gas viscosity $\mathrm{kg} \mathrm{m}^{-1} \mathrm{~s}^{-1}$

$\lambda \quad$ Mean free path of the gas, $m$

$\zeta \quad$ Volume fraction of aerosol material

$\rho \quad$ Mass density of the particles

$\Omega \quad$ Shape factor of size distribution

$\sigma_{g} \quad$ Geometric mean deviation of size distribution $\phi \quad$ Mean of IGD

$\varphi \quad$ Shape parameter of IGD

$\psi \quad$ Size distribution density function in SPSD

$\eta \quad$ Particle volume in the SPSD

$\tau \quad$ Scaled time

\section{c. Abbreviations}

KCE Kinetic coagulation equation

IGD Inverse Gaussian distribution

MOM Method of moments

SPSD Self-preserving size distribution

STM Similarity transformation method

TEMOM Taylor series expansion method of moments

IGDMOM Inverse Gaussian method of moments

Log MOM Lognormal method of moments

SM Sectional method

ODE Ordinary differential equation

SPT Self-preserving time

\section{REFERENCES}

Alexandrov, M. D., and A. A. Lacis, 2000: A new three-parameter cloud/aerosol particle size distribution based on the generalized inverse Gaussian density function. Appl. Math. Comput., 116, 153-165, https://doi.org/10.1016/S0096-3003(99)00201-5.

Anand, S., and Y. S. Mayya, 2011: A simplified approach for solving coagulation-diffusion equation to estimate atmospheric background particle number loading factors contributed by emissions from localized sources. Atmos. Environ., 45, 44884496, https://doi.org/10.1016/j.atmosenv.2011.05.016.

Bott, A., 2000: A flux method for the numerical solution of the stochastic collection equation: Extension to two-dimensional particle distributions. J. Atmos. Sci., 57, 284-294, https://doi.org/ 10.1175/1520-0469(2000)057<0284:AFMFTN>2.0.CO;2.

Cai, C., and Coauthors, 2016: Incorporation of new particle formation and early growth treatments into WRF/Chem: Model improvement, evaluation, and impacts of anthropogenic aerosols over East Asia. Atmos. Environ., 124, 262-284, https://doi.org/10.1016/j.atmosenv.2015.05.046. 
Chen, Z., J. Lin, and M. Yu, 2014: Asymptotic behavior of the Taylor-expansion method of moments for solving a coagulation equation for Brownian particles. Particuology, 14, 124129, https://doi.org/10.1016/j.partic.2013.04.012.

Chhikara, R. S., and J. L. Folks, 1977: The inverse Gaussian distribution as a lifetime model. Technometrics, 19, 461-468, https://doi.org/10.1080/00401706.1977.10489586.

Clark, W. E., and K. T. Whitby, 1967: Concentration and size distribution measurements of atmospheric aerosols and a test of the theory of self-preserving size distributions. J. Atmos. Sci., 24, 677-687, https://doi.org/10.1175/1520-0469(1967)024<0677: CASDMO $>2.0 . \mathrm{CO} ; 2$.

Dekkers, P. J., and S. K. Friedlander, 2002: The self-preserving size distribution theory. I. Effects of the Knudsen number on aerosol agglomerate growth. J. Colloid Interface Sci., 248, 295305, https://doi.org/10.1006/jcis.2002.8212.

Ensor, D. S., 2011: Aerosol Science and Technology: History and Reviews. 1st ed. RTI Press, 584 pp.

Foitzik, L., 1965: The spectral extinction of the atmospheric aerosol by Mie particles with different Gaussian distributions. Gerlands Beitr. Geophys., 74, 198-206.

Folks, J. L., and R. S. Chhikara, 1978: The inverse Gaussian distribution and its statistical application-A review. J. Roy. Stat. Soc., 40B, 263-275, https://doi.org/10.1111/j.2517-6161.1978.tb01039.x.

Frenklach, M., 1985: Dynamics of discrete distribution for Smoluchowski coagulation model. J. Colloid Interface Sci., 108, 237-242, https://doi.org/10.1016/0021-9797(85)90256-5.

Friedlander, S. K., 1960: Similarity considerations for the particle-size spectrum of a coagulating, sedimenting aerosol. J. Meteor., 17, 479-483, https://doi.org/10.1175/15200469(1960)017<0479:SCFTPS > 2.0.CO;2.

_ 1961: Theoretical considerations for the particle size spectrum of stratospheric aerosol. J. Meteor., 18, 753-759, https:// doi.org/10.1175/1520-0469(1961)018<0753:TCFTPS >2.0.CO;2.

- 2000: Smoke, Dust and Haze: Fundamentals of Aerosol Dynamics. 2nd ed. Oxford University Press, 407 pp.

— , and C. S. Wang, 1966: The self-preserving particle size distribution for coagulation by Brownian motion. J. Colloid Interface Sci., 22, 126-132, https://doi.org/10.1016/0021-9797(66)90073-7.

Goudeli, E., M. L. Eggersdorfer, and S. E. Pratsinis, 2015: Coagulation-agglomeration of fractal-like particles: Structure and self-preserving size distribution. Langmuir, 31, 1320-1327, https://doi.org/10.1021/la504296z.

Hidy, G. M., 1965: On the theory of the coagulation of noninteracting particles in Brownian motion. J. Colloid Sci., 20, 123144, https://doi.org/10.1016/0095-8522(65)90003-6.

— netics of coagulation. J. Colloid Sci., 20, 867-874, https:// doi.org/10.1016/0095-8522(65)90059-0.

Hussein, T., K. Hämeri, P. Aalto, A. Asmi, L. Kakko, and M. Kulmala, 2004: Particle size characterization and the indoor-to-outdoor relationship of atmospheric aerosols in Helsinki. Scand. J. Work Environ. Health, 30, 54-62, https:// www.sjweh.fi/show_abstract.php?abstract_id $=815$.

- - and Coauthors, 2020: Indoor particle concentrations, size distributions, and exposures in Middle Eastern microenvironments. Atmosphere, 11, 41, https://doi.org/10.3390/ atmos11010041.

Junge, C. E., 1969: Comments on "Concentration and size distribution measurements of atmospheric aerosols and a test of the theory of self-preserving size distributions." J. Atmos. Sci., 26, 603-608, https://doi.org/10.1175/1520-0469(1969)026<0603: COASDM $>2.0 . \mathrm{CO} ; 2$.
Kelesidis, G. A., and E. Goudeli, 2021: Self-preserving size distribution and collision frequency of flame-made nanoparticles in the transition regime. Proc. Combust. Inst., 38, 1233-1240, https://doi.org/10.1016/j.proci.2020.07.147.

Khvorostyanov, V. I., and J. A. Curry, 2008: Analytical solutions to the stochastic kinetic equation for liquid and ice particle size spectra. Part II: Large-size fraction in precipitating clouds. J. Atmos. Sci., 65, 2044-2063, https://doi.org/10.1175/ 2007JAS2485.1.

Klett, J. D., 1975: Class of solutions to the steady-state, sourceenhanced, kinetic coagulation equation. J. Atmos. Sci., 32, 380-389, https://doi.org/10.1175/1520-0469(1975)032<0380: ACOSTT $<2.0 . C O ; 2$.

Kolmogorov, A. N., 1941: On the log-normal law of particle size distribution during crushing. Dokl. Akad. Nauk SSSR, 31, 99-101.

Kreidenweis, S. M., M. Petters, and U. Lohmann, 2019: 100 years of progress in cloud physics, aerosols, and aerosol chemistry research. A Century of Progress in Atmospheric and Related Sciences: Celebrating the American Meteorological Society Centennial, Meteor. Monogr., No. 59, Amer. Meteor. Soc., https://doi.org/10.1175/AMSMONOGRAPHS-D-18-0024.1.

Lai, F. S., S. K. Friedlander, J. Pich, and G. M. Hidy, 1972: The selfpreserving particle size distribution for Brownian coagulation in the free-molecule regime. J. Colloid Interface Sci., 39, 395405, https://doi.org/10.1016/0021-9797(72)90034-3.

Landgrebe, J. D., and S. E. Pratsinis, 1990: A discrete sectional model for particulate production by gas phase chemical reaction and aerosol coagulation in the free molecular regime. J. Colloid Interface Sci., 139, 63-86, https://doi.org/10.1016/ 0021-9797(90)90445-T.

Lee, K. W., 1983: Change of particle-size distribution during Brownian coagulation. J. Colloid Interface Sci., 92, 315-325, https://doi.org/10.1016/0021-9797(83)90153-4.

_, H. Chen, and J. A. Gieseke, 1984: Log-normally preserving size distribution for Brownian coagulation in the freemolecule regime. Aerosol Sci. Technol., 3, 53-62, https:// doi.org/10.1080/02786828408958993.

- L. A. Curtis, and H. Chen, 1990: An analytic solution to free molecule aerosol coagulation. Aerosol Sci. Technol., 12, 457462, https://doi.org/10.1080/02786829008959360.

— Y Y. J. Lee, and D. S. Han, 1997: Analytic solution to low Knudsen number Brownian coagulation. J. Aerosol Sci., 28, S639-S640, https://doi.org/10.1016/S0021-8502(97)85319-0.

Li, X., A. Brandenburg, G. Svensson, N. Haugen, B. Mehlig, and I. Rogachevskii, 2020: Condensational and collisional growth of cloud droplets in a turbulent environment. J. Atmos. Sci., 77, 337-353, https://doi.org/10.1175/JAS-D-19-0107.1.

Liu, B. Y. H., and K. T. Whitby, 1968: Dynamic equilibrium in selfpreserving aerosols. J. Colloid Interface Sci., 26, 161-165, https://doi.org/10.1016/0021-9797(68)90308-1.

Long, A. B., 1974: Solutions to the droplet collection equation for polynomial kernels. J. Atmos. Sci., 31, 1040-1052, https://doi.org/ 10.1175/1520-0469(1974)031<1040:STTDCE > 2.0.CO;2.

Müller, H., 1928: Zur allgemeinen Theorie ser raschen Koagulation. Kolloid Chem. Beih., 27, 223-250.

Nathans, M. W., R. Thews, W. D. Holland, and P. A. Benson, 1970: Particle size distribution in clouds from nuclear airbursts. J. Geophys. Res., 75, 7559-7572, https://doi.org/ 10.1029/JC075i036p07559.

Otto, E., H. Fissan, S. Park, and K. Lee, 1999: The log-normal size distribution theory of Brownian aerosol coagulation for the entire particle size range: Part II-Analytical solution using 
Dahneke's coagulation kernel. J. Aerosol Sci., 30, 17-34, https://doi.org/10.1016/S0021-8502(98)00038-X.

Park, S., and K. Lee, 2001: Asymptotic particle size distributions attained during coagulation processes. J. Colloid Interface Sci., 233, 117-123, https://doi.org/10.1006/jcis.2000.7222.

_- F. Kruis, K. Lee, and H. Fissan, 2000: Evolution of particle size distribution due to turbulent coagulation. J. Aerosol Sci., 31, 572-573, https://doi.org/10.1016/S0021-8502(00)90583-4.

Pratsinis, S. E., 1988: Simultaneous nucleation, condensation, and coagulation in aerosol reactors. J. Colloid Interface Sci., 124, 416-427, https://doi.org/10.1016/0021-9797(88)90180-4.

Pruppacher, H. R., and J. D. Klett, 1997: Microphysics of Clouds and Precipitation. Springer Netherlands, $954 \mathrm{pp}$.

Riemer, N., M. West, R. Zaveri, and R. C. Easter, 2009: Simulating the evolution of soot mixing state with a particle-resolved aerosol model. J. Geophys. Res., 114, D09202, https://doi.org/10.1029/2008JD011073.

Schrödinger, E., 1915: Zur theorie der fall-und steigversuche an teilchen mit Brownscher bewegung. Phys. Z., 16, 289-295.

Scott, W. T., 1968: Analytic solutions of cloud droplet coalescence I. J. Atmos. Sci., 25, 54-65, https://doi.org/10.1175/15200469(1968)025<0054:ASOCDC > 2.0.CO;2.

Seshadri, V., 1999: The Inverse Gaussian Distribution: Statistical Theory and Applications. Springer, 347 pp.

Shen, J., M. Yu, T. L. Chan, C. Tu, and Y. Liu, 2020a: Efficient method of moments for simulating atmospheric aerosol growth: Model description, verification, and application. J. Geophys. Res. Atmos., 125, e2019JD032172, https://doi.org/10.1029/2019JD032172.

—_, _- and J. Lin, 2020b: Description of atmospheric aerosol dynamics using an inverse Gaussian distributed method of moments. J. Atmos. Sci., 77, 3011-3031, https:// doi.org/10.1175/JAS-D-20-0077.1.

Smoluchowski, M. V., 1915: Notiz uiber die Berechnung der Brownschen Molekularbewegung bei der EhrenhaftMillikanschen Versuchsanordning. Phys. Z., 16, 318-321.

__, 1917: Versuch einer mathematischen Theorie der Koagulationskinetik kolloider Lösungen. Z. Phys. Chem., 21, 98-104.

Söderlund, J., L. B. Kiss, G. A. Niklasson, and C. G. Granqvist, 1998: Lognormal size distributions in particle growth processes without coagulation. Phys. Rev. Lett., 80, 2386-2388, https://doi.org/10.1103/PhysRevLett.80.2386.

Swift, D. L., and S. K. Friedlander, 1964: The coagulation of hydrosols by Brownian motion and laminar shear flow. J. Colloid Sci., 19, 621-647, https://doi.org/10.1016/0095-8522(64)90085-6.

Turco, R. P., and F. Yu, 1997: Aerosol invariance in expanding coagulating plume. Geophys. Res. Lett., 24, 1223-1226, https:// doi.org/10.1029/97GL01092.

— , and - 1998: Aerosol size distribution in a coagulating plume: Analytical behavior and modeling applications. Geophys. Res. Lett., 25, 927-930, https://doi.org/10.1029/98GL00324.

— , and — 1999: Particle size distributions in an expanding plume undergoing simultaneous coagulation and condensation. J. Geophys. Res., 104, 19227-19241, https://doi.org/ 10.1029/1999JD900321.

Tweedie, M. C. K., 1957: Statistical properties of inverse Gaussian distributions. I. Ann. Math. Stat., 28, 362-377, https://doi.org/ 10.1214/aoms/1177706964.

Valioulis, I., and E. J. List, 1984: A numerical evaluation of the stochastic completeness of the kinetic coagulation equation. J. Atmos. Sci., 41, 2516-2530, https://doi.org/10.1175/15200469(1984)041<2516:ANEOTS>2.0.CO;2.

Vemury, S., K. Kusters, and S. Pratsinis, 1994: Time-lag for attainment of the self-preserving particle size distribution by coagulation. J. Colloid Interface Sci., 165, 53-59, https:// doi.org/10.1006/jcis.1994.1204.

Wang, C. S., and S. K. Friedlander, 1967: The self-preserving particle size distribution for coagulation by Brownian motion II. Small particle slip correction and simultaneous shear flow. J. Colloid Interface Sci., 24, 170-179, https://doi.org/10.1016/ 0021-9797(67)90215-9.

Wang, J., Q. Zhao, S. Cui, and C. Zhu, 2012: Assessment of aerosol modes used in the MODIS ocean aerosol retrieval. J. Atmos. Sci., 69, 3595-3605, https://doi.org/10.1175/JAS-D-12-051.1.

Wang, K., S. Yu, and W. Peng, 2019: A novel moment method using the log skew normal distribution for particle coagulation. J. Aerosol Sci., 134, 95-108, https://doi.org/10.1016/j.jaerosci.2019.04.013.

Wang, L. P., Y. Xue, and W. W. Grabowski, 2007: A bin integral method for solving the kinetic collection equation. J. Comput. Phys., 226, 59-88, https://doi.org/10.1016/j.jcp.2007.03.029.

Xie, M., and Q. He, 2013: Analytical solution of TEMOM model for particle population balance equation due to Brownian coagulation. J. Aerosol Sci., 66, 24-30, https://doi.org/10.1016/ j.jaerosci.2013.08.006.

Yu, F., and R. P. Turco, 1998: The formation and evolution of aerosols in stratospheric aircraft plumes: Numerical simulations and comparisons with observations. J. Geophys. Res., 103, 25 915-25 934, https://doi.org/10.1029/98JD02453.

$\mathrm{Yu}$, M., and J. Lin, 2009a: Solution of the agglomerate Brownian coagulation using Taylor-expansion moment method. J. Colloid Interface Sci., 336, 142-149, https:// doi.org/10.1016/j.jcis.2009.03.030.

$\longrightarrow$, and $-2009 \mathrm{~b}$ : Taylor-expansion moment method for agglomerate coagulation due to Brownian motion in the entire size regime. J. Aerosol Sci., 40, 549-562, https://doi.org/ 10.1016/j.jaerosci.2009.03.001.

— and - 2017: New scheme for implementing the method of moments with interpolative closure. Aerosol Sci. Technol., 51, 956-971, https://doi.org/10.1080/02786826.2017.1319566.

- , and 2018: Taylor series expansion scheme applied for solving population balance equation. Rev. Chem. Eng., 34, 561-594, https://doi.org/10.1515/revce-2016-0061.

,-- , and T. Chan, 2008: A new moment method for solving the coagulation equation for particles in Brownian motion. Aerosol Sci. Technol., 42, 705-713, https://doi.org/10.1080/ 02786820802232972.

_ _ - J. Cao, and M. Seipenbusch, 2015a: An analytical solution for the population balance equation using a moment method. Particuology, 18, 194-200, https://doi.org/10.1016/ j.partic.2014.06.006.

$\longrightarrow,-$ _ X. Zhang, G. Jin, J. Lin, and M. Seipenbusch, 2015b: A new analytical solution for solving the population balance equation in the continuum-slip regime. J. Aerosol Sci., 80, 110, https://doi.org/10.1016/j.jaerosci.2014.10.007.

, Y. Liu, G. Jin, and H. Jin, 2016: A new analytical solution for agglomerate growth undergoing Brownian coagulation. Appl. Math. Model., 40, 5497-5509, https://doi.org/ 10.1016/j.apm.2016.01.009.

,-- , and A. J. Koivisto, 2017: An efficient algorithm scheme for implementing the TEMOM for resolving aerosol dynamics. Aerosol Sci. Eng., 1, 119-137, https://doi.org/10.1007/ s41810-017-0012-2.

Zhao, Z., J. C. Crittenden, H. Zhao, and Y. Chen, 2015: The selfpreserving size distribution of fractal aggregates coagulating by Brownian motion and simultaneous fluid shear at low Peclet numbers: Numerical solutions. J. Aerosol Sci., 87, 1-16, https:// doi.org/10.1016/j.jaerosci.2015.04.006. 